\title{
Duality and Hidden Symmetries in Interacting Particle Systems
}

\author{
Cristian Giardinà · Jorge Kurchan · Frank Redig • \\ Kiamars Vafayi
}

Received: 4 February 2009 / Accepted: 28 February 2009 / Published online: 14 March 2009

(C) The Author(s) 2009. This article is published with open access at Springerlink.com

\begin{abstract}
In the context of Markov processes, both in discrete and continuous setting, we show a general relation between duality functions and symmetries of the generator. If the generator can be written in the form of a Hamiltonian of a quantum spin system, then the "hidden" symmetries are easily derived. We illustrate our approach in processes of symmetric exclusion type, in which the symmetry is of $S U(2)$ type, as well as for the KipnisMarchioro-Presutti (KMP) model for which we unveil its $S U(1,1)$ symmetry. The KMP model is in turn an instantaneous thermalization limit of the energy process associated to a large family of models of interacting diffusions, which we call Brownian energy process (BEP) and which all possess the $S U(1,1)$ symmetry. We treat in details the case where the system is in contact with reservoirs and the dual process becomes absorbing.
\end{abstract}

Keywords Non-equilibrium statistical mechanics · Interacting particle systems · Duality

\section{Introduction}

Duality is a technique developed in the probabilistic literature that allows to obtain elegant and general solutions of some problems in interacting particle systems. One transforms the

\footnotetext{
C. Giardinà $(\bowtie)$

Department of Mathematics and Computer Science, Eindhoven University, P.O. Box 513, 5600 MB Eindhoven, The Netherlands

e-mail: c.giardina@tue.nl

J. Kurchan

CNRS-Ecole Supérieure de Physique et de Chimie Industrielles, rue Vauquelin 10, 75231 Paris, France

e-mail: jorge@pmmh.espci.fr

F. Redig $\cdot$ K. Vafayi

Mathematisch Instituut Universiteit Leiden, Niels Bohrweg 1, 2333 CA Leiden, The Netherlands

F. Redig

e-mail: redig@ math.leidenuniv.nl

K. Vafayi

e-mail: vafayi@math.leidenuniv.nl
} 
evaluation of a correlation function in the original model to a simpler quantity in the dual one.

The basic idea of duality in interacting particle systems goes back to Spitzer [17] who introduced it for symmetric exclusion process (SEP) and independent random walkers to characterize the stationary distribution. Later, Ligget [9] systematically introduced duality for spin systems and used it, among others, for the complete characterization of ergodic properties of SEP, voter model, etc. Duality property might also be useful in the context of transport models and non-equilibrium statistical mechanics, that is when the bulk particle systems is in contact at its boundaries with reservoirs working at different values of their parameters. For instance, considering again the symmetric exclusion process in contact with particle reservoirs at different chemical potentials, Spohn used duality to compute the 2-point correlation function [18], showing the existence of long-range correlations in non-equilibrium systems. In the case of energy transport, i.e. interacting particle systems with a continuous dynamical variable (the energy) connected at their boundaries to thermal reservoirs working at different temperatures, duality has been constructed for the KipnisMarchioro-Presutti (KMP) model [8] for heat conduction and also for other models [6]. Consequences of duality include the possibility to express the $n$-point energy correlation functions in terms of $n$ (interacting) random walkers. Duality has also been used in the study of biological population models, see [10] and references therein.

One should notice that the construction of a dual process is usually performed with an ad-hoc procedure which requires the ansatz of a proper duality function on which the duality property can be established. The closure of $n$-point correlations functions at each order might be an indication that a dual process exists. However in the general case the closure property is neither sufficient nor necessary to construct the dual process. In this paper we present a general procedure to derive a duality function and a dual process from the symmetries of the original process. When applied to transport models, our theorems allow to identify the source of the existence of a dual process with the non-abelian symmetries of the evolution operator. The idea is simple: transport models have in the bulk a symmetry associated with a conserved quantity, the one that is transported. It may happen in some cases that this symmetry is a subgroup of a larger group, i.e. that extra (less obvious) symmetry are present. In that case, one can describe the same physical situation as the transport of another quantity (another element of the group), and in some cases this makes the problem simpler. In the physics literature Sandow and Schutz [13] realized that this is case for the SEP process, whose $S U(2)$ symmetry they made explicit by writing the evolution operator in quantum spin notation. In this paper we study in full generality the relation between duality and symmetries. ${ }^{1}$

We shall give a general scheme to construct a dual stochastic process for continuous time Markov processes whose generator has a symmetry. After the introduction of the general setting we present the main theorems describing the construction of a dual process. To make them explicit we then analyze a series of simple examples involving systems with only two sites. These examples cover well-know cases, like the independent random walkers case, and new stochastic processes as well.

We shall be mainly interested in applications to interacting particle systems describing transport of mass and/or energy. As for transport of particles, we study exclusion processes.

\footnotetext{
${ }^{1}$ We will restrict to the notion of symmetry groups and all the examples in the present paper are Lie algebras with associated Lie groups. We mention that one could also consider duality relations in the case of a quantum algebra, which a much more richer and complicated structure than a Lie algebra. For relevant example see $[11,12,14]$.
} 
The results of the section on exclusion processes partly overlap with [13]. We study indeed the same generalized version of exclusion processes with partial exclusion, i.e. each site can accommodate up to $2 j$ particles, with $j \in \mathbb{N} / 2$. We give however a different perspective: the model is seen as the process that is obtained when one considers the standard exclusion process on a ladder graph with $2 j$ layers and then one looks at the number of particles on each site, ignoring the layer to which they belong. We exhibit new duality functions, which are naturally constructed in our approach, and we study with full details the case where the system is in contact with reservoirs.

For energy transport models we study a large class of diffusive models which we call Brownian energy process (BEP). They all possess the $S U(1,1)$ symmetry and generalize the model that was introduced in [6]. The family of models is labeled by the spin value $k \in \mathbb{N} / 4$, with the case in [6] corresponding to $k=1 / 4$. For the whole class of models, we show how to construct the dual process from the $S U(1,1)$ symmetry. We prove that the case with $k=1 / 2$ is related to the KMP model, thus explaining the construction of the dual process in that context.

\section{Definitions and Results}

\subsection{Generalities}

Let $\left(\eta_{t}\right)_{t \geq 0}$ denote a Markov process on a state space $\Omega$. Elements of the state space are denoted by $\eta, \xi, \zeta, \ldots$ The probability measure on path space starting from $\eta$ is called $\mathbb{P}_{\eta}$, and $\mathbb{E}_{\eta}$ denotes expectation with respect to $\mathbb{P}_{\eta}$. In the whole of this paper, we will restrict to Feller processes. In that case, to the process $\left(\eta_{t}\right)_{t \geq 0}$ there corresponds a strongly continuous, positivity-preserving, contraction semigroup $A_{t}: \mathcal{C}(\Omega) \rightarrow \mathcal{C}(\Omega)$, the domain of which is the set $\mathcal{C}(\Omega)$ of continuous functions $f: \Omega \rightarrow \mathbb{R}$,

$$
A_{t} f(\eta):=\mathbb{E}_{\eta} f\left(\eta_{t}\right)=\mathbb{E}\left(f\left(\eta_{t}\right) \mid \eta_{0}=\eta\right)=\int f\left(\eta^{\prime}\right) p_{t}\left(\eta, d \eta^{\prime}\right)
$$

where $p_{t}\left(\eta, d \eta^{\prime}\right)$ is the transition kernel of the process. The infinitesimal generator of the semigroup is denoted by $L$,

$$
L f=\lim _{t \rightarrow 0} \frac{A_{t} f-f}{t}
$$

and is defined on its natural domain, i.e. the set of functions $f: \Omega \rightarrow \mathbb{R}$ for which the limit in the r.h.s. exists in the uniform metric. We also consider the adjoint of the semigroup, with domain $\mathcal{M}(\Omega)$ the set of signed finite Borel measures, $A_{t}^{*}: \mathcal{M}(\Omega) \rightarrow \mathcal{M}(\Omega)$, defined by

$$
\left\langle f, A_{t}^{*} \mu\right\rangle=\left\langle A_{t} f, \mu\right\rangle
$$

where the pairing $\langle\cdot, \cdot\rangle: \mathcal{C}(\Omega) \times \mathcal{M}(\Omega) \rightarrow \mathbb{R}$ is given by

$$
\langle f, \mu\rangle=\int f d \mu
$$

The processes which appear in our applications will always be either jump process or diffusions. 
Example 2.1 In the case that the Markov process $\left(\eta_{t}\right)_{t \geq 0}$ is a pure jump process and the state space $\Omega$ is finite or countable then the generator is of the form

$$
L f(\eta)=\sum_{\eta^{\prime} \in \Omega} c\left(\eta, \eta^{\prime}\right)\left(f\left(\eta^{\prime}\right)-f(\eta)\right)
$$

where $c\left(\eta, \eta^{\prime}\right) \geq 0$ is the rate for a transition from configuration $\eta$ to configuration $\eta^{\prime}$. Equivalently we can write

$$
L f(\eta)=\sum_{\eta^{\prime} \in \Omega} L\left(\eta, \eta^{\prime}\right) f\left(\eta^{\prime}\right)
$$

where $L\left(\eta, \eta^{\prime}\right)$ is a matrix having positive off-diagonal elements and rows sum equal to zero, namely

$$
L\left(\eta, \eta^{\prime}\right)= \begin{cases}c\left(\eta, \eta^{\prime}\right) & \text { if } \eta \neq \eta^{\prime} \\ -\sum_{\eta^{\prime \prime} \neq \eta} c\left(\eta, \eta^{\prime \prime}\right) & \text { if } \eta=\eta^{\prime}\end{cases}
$$

In the context of a countable state space $\Omega$ we have the usual exponential of a matrix, so that

$$
A_{t}=e^{t L}=\sum_{i=0}^{\infty}(t L)^{i} / i !
$$

and $A_{t}^{*}=A_{t}^{T}$ where the superscript ${ }^{T}$ denotes transposition.

Example 2.2 General diffusion processes with state space $\Omega=\mathbb{R}^{N}$ are also considered here. In this case the generator take the form of a differential operator of the second order

$$
L f=\sum_{i, j=1}^{N} a\left(x_{i}, x_{j}\right) \frac{\partial^{2} f}{\partial x_{i} \partial x_{j}}+\sum_{i=1}^{N} b\left(x_{i}\right) \frac{\partial f}{\partial x_{i}}
$$

(see [19] for general conditions which guarantees that $L$ satisfy the maximum principle and thus generate a positivity preserving semigroup).

\subsection{Duality and Self-duality}

Definition 2.3 (Self-duality) Consider two independent copies $\left(\eta_{t}\right)_{t \geq 0}$ and $\left(\xi_{t}\right)_{t \geq 0}$ of a continuous time Markov processes on a state space $\Omega$. We say that the process is self-dual with self-duality function $D: \Omega \times \Omega \rightarrow \mathbb{R}$ if for all $(\eta, \xi) \in \Omega \times \Omega$, we have

$$
\mathbb{E}_{\eta} D\left(\eta_{t}, \xi\right)=\mathbb{E}_{\xi} D\left(\eta, \xi_{t}\right)
$$

Definition 2.4 (Duality) Consider two continuous time Markov processes: $\left(\eta_{t}\right)_{t>0}$ on a state space $\Omega$ and $\left(\xi_{t}\right)_{t \geq 0}$ on a state space $\Omega_{\text {dual }}$. We say that $\left(\xi_{t}\right)_{t \geq 0}$ is the dual of $\left(\eta_{t}\right)_{t \geq 0}$ with duality function $D: \Omega \times \Omega_{\text {dual }} \rightarrow \mathbb{R}$ if for all $\eta \in \Omega, \xi \in \Omega_{\text {dual }}$ we have

$$
\mathbb{E}_{\eta} D\left(\eta_{t}, \xi\right)=\mathbb{E}_{\xi}^{\text {dual }} D\left(\eta, \xi_{t}\right)
$$

If $A_{t}$ denotes the semigroup of the original process $\left(\eta_{t}\right)_{t \geq 0}$ and $A_{t}^{\text {dual }}$ denotes the semigroup of the related dual process $\left(\xi_{t}\right)_{t \geq 0}$ then, using (1), the Definition 2.4 is equivalent to

$$
A_{t} D(\eta, \xi)=A_{t}^{\text {dual }} D(\eta, \xi)
$$


where it is understood that on the 1.h.s. of (4) the operator $A_{t}$ works on the $\eta$ variable, while on the r.h.s. the operator $A_{t}^{\text {dual }}$ works on the $\xi$ variable.

If the original process $\left(\eta_{t}\right)_{t \geq 0}$ and the dual process $\left(\xi_{t}\right)_{t \geq 0}$ are Markov processes with finite or countably infinite state space $\Omega$, resp. $\Omega_{\text {dual }}$ (cfr. Example 2.1) property (4) is equivalent with its "infinitesimal version" in terms of the generators

$$
\sum_{\eta^{\prime} \in \Omega} L\left(\eta, \eta^{\prime}\right) D\left(\eta^{\prime}, \xi\right)=\sum_{\xi^{\prime} \in \Omega} L_{\text {dual }}\left(\xi, \xi^{\prime}\right) D\left(\eta, \xi^{\prime}\right)
$$

In matrix notation, this reads

$$
L D=D L_{\text {dual }}^{T}
$$

where $D$ is the matrix with elements $D(\eta, \xi)$ and $(\eta, \xi) \in \Omega \times \Omega_{\text {dual }}$. Remark that in this case $D$ is not necessarily a square matrix, because the state spaces $\Omega$ and $\Omega_{\text {dual }}$ are not necessarily equal and or of equal cardinality.

When $\Omega=\Omega_{\text {dual }}$ and $A_{t}=A_{t}^{\text {dual }}$, then an equivalent condition for self-duality (cfr. (2)) is

$$
L D=D L^{T}
$$

\subsection{Duality and Symmetries}

We first discuss self-duality and then duality. We consider the simple context of finite or countably infinite state space Markov processes. In many cases of interacting particle systems, the generator is a sum of operators working only on a finite set of coordinates of the configuration. Therefore, showing (self)-duality reduces to showing (self)-duality for the individual terms appearing in this sum, which is a finite state space situation.

Definition 2.5 Let $A$ and $B$ be two matrices having the same dimension. We say that $A$ is a symmetry of $B$ if $A$ commutes with $B$, i.e.

$$
A B=B A
$$

The first theorem shows that self-duality functions and symmetries are in one-to-one correspondence, provided $L$ and $L^{T}$ are similar matrices, which is automatically the case in the finite state space context (see also [15, 16], (5.7)).

Theorem 2.6 Let $L$ be the generator of a finite or countable state space Markov process. Let $Q$ be a matrix such that

$$
L^{T}=Q L Q^{-1}
$$

Then we have

1. If $S$ is a symmetry of the generator, then $S Q^{-1}$ is a self-duality function.

2. If $D$ is a self-duality function, then $D Q$ is a symmetry of the generator.

3. If $S$ is a symmetry of $L^{T}$, then $Q^{-1} S$ is a self-duality function.

4. If $D$ is a self-duality function, then $Q D$ commutes with $L^{T}$.

Proof The proof is elementary. We show items 1 and 2 (items 3 and 4 are obtained in a similar manner). Combining (9) with (8), we find

$$
L\left(S Q^{-1}\right)=\left(S Q^{-1}\right) L^{T}
$$


i.e., $D=S Q^{-1}$ is a self-duality function (see (7)). Conversely, if $D$ is a self-duality function, then combining (9) with (7) one proves (8) for $S=D Q$.

Remark 2.7 Self-duality functions are not unique, i.e. there might exist several self-duality functions for a process. This is evident form the fact that if $D$ is a duality function for self-duality, and $S$ is a symmetry, then $S D$ is also a duality function for self-duality. An interesting question is to study the vector space of self-duality functions, its dimension, etc. However this question is not addressed in this paper. See [10] for a discussion of this issue and some examples in the context of Markov processes with discrete state space.

Remark 2.8 In the finite state space context, $L$ and $L^{T}$ are always similar matrices [20], i.e., there exists a conjugation matrix $Q$ such that $L^{T}=Q L Q^{-1}$. In interacting particle system the matrix $Q$ can usually be easily constructed. As an example, in the case that $\mathcal{L}$ has a reversible measure, i.e., a probability measure $\mu$ on $\Omega$ such that

$$
\mu(\eta) L\left(\eta, \eta^{\prime}\right)=\mu\left(\eta^{\prime}\right) L\left(\eta^{\prime}, \eta\right)
$$

for all $\eta, \eta^{\prime} \in \Omega$, then a diagonal conjugation matrix $Q$ is given by

$$
Q\left(\eta, \eta^{\prime}\right)=\mu(\eta) \delta_{\eta, \eta^{\prime}}
$$

In general, if $\mu$ is a stationary measure then

$$
L_{r e v}(\eta, \xi):=\frac{L(\xi, \eta) \mu(\eta)}{\mu(\xi)}
$$

is the generator of the time-reversed process, which is clearly similar to $L^{T}$. Therefore, the similarity of $L$ and $L^{T}$ is equivalent with the similarity of the generator and the time-reversed generator.

Self-duality is a particular case of duality. To generalize Theorem 2.6 to the context of (general) duality we need the notion of conjugation between two matrices.

Definition 2.9 Let $A$ be a matrix of dimension $m \times m$ and let $B$ be a matrix of dimension $n \times n$. $A$ and $B$ are called conjugate if there exist matrices $C$ of dimension $m \times n$ and $\tilde{C}$ of dimension $n \times m$ such that

$$
A C=C B, \quad \tilde{C} A=B \tilde{C}
$$

We then have the following analogue of Theorem 2.6.

Theorem 2.10 Let $L$ and $L_{\text {dual }}$ be generators of finite or countable state space Markov chains. Then we have the following.

1. If $Q$ is the matrix that gives the similarity

$$
L_{\text {dual }}^{T}=Q L_{\text {dual }} Q^{-1}
$$

and $C$ and $\tilde{C}$ are the matrices giving the conjugacy between $L$ and $L_{\text {dual }}$ in the sense of Definition 2.9, then: 
(a) For any symmetry $S$ of the generator $L, D=S C Q^{-1}$ is a duality function.

(b) If $D$ is a duality function, then $S=D Q \tilde{C}$ is a symmetry of $L$.

2. If $Q$ is the matrix that gives the similarity

$$
L^{T}=Q L Q^{-1}
$$

and $C$ and $\tilde{C}$ are the matrices giving the conjugacy between $L^{T}$ and $L_{\text {dual }}^{T}$ in the sense of Definition 2.9, then:

(a) For any symmetry $S$ of the transposed generator $L^{T}, Q^{-1} S C$ is a duality function.

(b) If $D$ is a duality function, then $Q D \tilde{C}$ commutes with $L^{T}$.

Proof The proof of item 1(a) is given by the following series of equalities

$$
L\left(S C Q^{-1}\right)=S L C Q^{-1}=S C L_{\text {dual }} Q^{-1}=\left(S C Q^{-1}\right) L_{\text {dual }}^{T}
$$

The first equality uses the hypothesis of $S$ being a symmetry of the generator $L$, the second comes from the conjugation of the generators, the third is obtained from the similarity transformation (14). If one recall (6) then (16) shows that $D=S C Q^{-1}$ is a duality function. The proof of the other items follow from a similar argument.

\section{Examples with Two Sites}

In this section we present a series of examples where particles jump on two lattice sites. We wish to show how (self)-duality can be established by making use of the previous theorems. To identify the symmetries we will rewrite the stochastic generator, or its adjoint, in terms of generators of some symmetry group. Some of the examples will be useful later for the study of transport models. In fact, many transport models such as the exclusion process have a generator that is written as the sum of operators working on two sites.

\subsection{Self-duality for Symmetric Exclusion}

We first recover the classical self-duality for symmetric exclusion [7, 9]. One has two sites (labeled 1,2) and configurations have at most one particle at each site. Particles hop at rate one from one site to another, and jumps leading to more than one particle at a site are suppressed. As usual we write 0,1 for absence resp. presence of particle. The state space is then $\Omega=\{00,01,10,11\}$. Elements in the state space are denoted as $\eta=\left(\eta_{1} \eta_{2}\right)$. The matrix elements of the generator are given by $L_{01,10}=L_{10,01}=1=-L_{01,01}=-L_{10,10}$, and all other elements are zero.

To apply Theorem 2.6 we need to identify a symmetry $S$ of the generator. The transposed of the generator can be written as

$$
L^{T}=J_{1}^{+} \otimes J_{2}^{-}+J_{1}^{-} \otimes J_{2}^{+}+2 J_{1}^{0} \otimes J_{2}^{0}-\frac{1}{2} \mathbb{1}_{1} \otimes \mathbb{1}_{2}
$$

where the operators $J_{i}^{a}$ with $i \in\{1,2\}$ and $a \in\{+,-, 0\}$ act on a 2-dimensional Hilbert space, with basis $|0\rangle=\left(\begin{array}{l}1 \\ 0\end{array}\right),|1\rangle=\left(\begin{array}{l}0 \\ 1\end{array}\right)$, as

$$
J_{i}^{+}=\left(\begin{array}{cc}
0 & 0 \\
1 & 0
\end{array}\right), \quad J_{i}^{-}=\left(\begin{array}{ll}
0 & 1 \\
0 & 0
\end{array}\right), \quad J_{i}^{0}=\left(\begin{array}{cc}
-1 / 2 & 0 \\
0 & 1 / 2
\end{array}\right)
$$


and $\mathbb{1}_{i}$ is the identity matrix. The operators $J_{i}^{a}$ with $a \in\{+,-, 0\}$ satisfy the $S U(2)$ commutation relations:

$$
\begin{aligned}
{\left[J_{i}^{0}, J_{i}^{ \pm}\right] } & = \pm J_{i}^{ \pm} \\
{\left[J_{i}^{-}, J_{i}^{+}\right] } & =-2 J_{i}^{0}
\end{aligned}
$$

from which we deduce (cfr. (17)) that $L^{T}$ commutes with the three generators of the $S U$ (2) group, $J^{a}=J_{1}^{a} \otimes \mathbb{1}_{2}+\mathbb{1}_{1} \otimes J_{2}^{a}$ for $a \in\{+,-, 0\}$. A possible choice for the symmetry of $L^{T}$ is then obtained by considering the creation operator $J^{+}$and exponentiating in order to have a factorized form

$$
S=e^{J^{+}}=e^{J_{1}^{+} \otimes \mathbb{1}_{2}+\mathbb{1}_{1} \otimes J_{2}^{+}}=e^{J_{1}^{+}} \otimes e^{J_{2}^{+}}=S_{1} \otimes S_{2}
$$

More explicitly, in the basis $|0\rangle \otimes|0\rangle,|0\rangle \otimes|1\rangle,|1\rangle \otimes|0\rangle,|1\rangle \otimes|1\rangle$, the matrix $S$ is

$$
S=\left(\begin{array}{ll}
1 & 0 \\
1 & 1
\end{array}\right) \otimes\left(\begin{array}{ll}
1 & 0 \\
1 & 1
\end{array}\right)=\left(\begin{array}{llll}
1 & 0 & 0 & 0 \\
1 & 1 & 0 & 0 \\
1 & 0 & 1 & 0 \\
1 & 1 & 1 & 1
\end{array}\right)
$$

We also need the similarity transformation between $L$ and $L^{T}$. The matrix $Q$, relating $L$ to its transposed, is the identity since $L$ is symmetric. A duality function for self-duality is thus given by $D=Q^{-1} S=S$. Notice that $D$ can also be written as

$$
D\left(\eta_{1} \eta_{2}, \xi_{1} \xi_{2}\right)=\prod_{i \in\{1,2\}: \xi_{i}=1} \eta_{i}
$$

which is the usual self-duality function of [9].

\subsection{Self-duality for $2 j$-symmetric Exclusion}

Now we consider two sites with at most $2 j$ particles on each site, with $j \in \mathbb{N} / 2$ (see also [13]). The state space is $\Omega=\Omega_{1} \times \Omega_{2}$ where $\Omega_{i}=\{0,1, \ldots, 2 j\}$. The rates for transitions are the following: if there are $\eta_{1}$ particles at site 1 and $\eta_{2}$ particles at site 2, a particle is moved from 1 to 2 at rate $\eta_{1}\left(2 j-\eta_{2}\right)$ and from 2 to 1 at rate $\eta_{2}\left(2 j-\eta_{1}\right)$. So in this case the generator is given by

$$
\begin{aligned}
L\left(\eta_{1} \eta_{2}, \eta_{1}^{\prime} \eta_{2}^{\prime}\right)= & \eta_{1}\left(2 j-\eta_{2}\right) \delta_{\eta_{1}-1, \eta_{1}^{\prime}} \delta_{\eta_{2}+1, \eta_{2}^{\prime}}+\eta_{2}\left(2 j-\eta_{1}\right) \delta_{\eta_{1}+1, \eta_{1}^{\prime}} \delta_{\eta_{2}-1, \eta_{2}^{\prime}} \\
& -\left(\eta_{1}\left(2 j-\eta_{2}\right)+\eta_{2}\left(2 j-\eta_{1}\right)\right) \delta_{\eta_{1}, \eta_{1}^{\prime}} \delta_{\eta_{2}, \eta_{2}^{\prime}}
\end{aligned}
$$

The transposed of this generator can also be expressed as the scalar product between two spin operators satisfying the $S U(2)$ algebra, namely

$$
L^{T}=J_{1}^{+} \otimes J_{2}^{-}+J_{1}^{-} \otimes J_{2}^{+}+2 J_{1}^{0} \otimes J_{2}^{0}-2 j^{2} \mathbb{1}_{1} \otimes \mathbb{1}_{2}
$$

where the $J_{i}^{a}, i \in\{1,2\}$ and $a \in\{+,-, 0\}$, act on a $(2 j+1)$-dimensional Hilbert space with orthonormal basis $|0\rangle,|1\rangle, \ldots,|2 j\rangle$ as

$$
\begin{aligned}
J_{i}^{+}\left|\eta_{i}\right\rangle & =\left(2 j-\eta_{i}\right)\left|\eta_{i}+1\right\rangle \\
J_{i}^{-}\left|\eta_{i}\right\rangle & =\eta_{i}\left|\eta_{i}-1\right\rangle \\
J_{i}^{0}\left|\eta_{i}\right\rangle & =\left(\eta_{i}-j\right)\left|\eta_{i}\right\rangle
\end{aligned}
$$


The standard symmetric exclusion process of the previous section is recovered when $j=1 / 2$. Reasoning as above, a symmetry of the generator is

$$
S=S_{1} \otimes S_{2}=e^{J_{1}^{+}} \otimes e^{J_{2}^{+}}
$$

which has matrix elements $S\left(\eta_{1} \eta_{2}, \xi_{1} \xi_{2}\right)=S_{1}\left(\eta_{1}, \xi_{1}\right) S_{2}\left(\eta_{2}, \xi_{2}\right)$ with

$$
S_{i}\left(\eta_{i}, \xi_{i}\right)=\left\langle\eta_{i}\left|e^{J_{i}^{+}}\right| \xi_{i}\right\rangle=\left(\begin{array}{c}
2 j-\eta_{i} \\
\eta_{i}-\xi_{i}
\end{array}\right)
$$

where we adopt the convention $\left(\begin{array}{l}n \\ m\end{array}\right)=0$ for $m>n$.

To detect the matrix $Q$ giving the similarity transform between $L$ and $L^{T}$ (notice that $L$ is not symmetric anymore for $j \neq 1 / 2$ ) we make use of Remark 2.8 and use the fact that the invariant measures of the $2 j$-symmetric exclusion process are products of binomials $\operatorname{Bin}(2 j, \rho)$, with a free parameter $0<\rho<1$ (this will be proved in Theorem 4.2). Therefore, if we choose $\rho=1 / 2$ then a possible choise is $Q=Q_{1} \otimes Q_{2}$ with

$$
Q_{i}\left(\eta_{i}, \eta_{i}^{\prime}\right)=\delta_{\eta_{i}, \eta_{i}^{\prime}}\left(\begin{array}{c}
2 j \\
\eta_{i}
\end{array}\right)
$$

Combining (22) and (23), Theorem 2.6 then implies that a duality function for self-duality is given by

$$
D=D_{1} \otimes D_{2}=Q_{1}^{-1} S_{1} \otimes Q_{2}^{-1} S_{2}
$$

with

$$
D_{i}\left(\eta_{i}, \xi_{i}\right)=\left(Q_{i}^{-1} S_{i}\right)\left(\eta_{i}, \xi_{i}\right)=\frac{\left(\begin{array}{l}
\eta_{i} \\
\xi_{i}
\end{array}\right)}{\left(\begin{array}{c}
2 J \\
\xi_{i}
\end{array}\right)}
$$

Later, in Theorem 4.2, we will give a probabilistic interpretation of this function.

\subsection{Self-duality for the Dual-BEP}

This is a process that can be viewed as a "bosonic" analogue of the SEP (particles attract each other rather than repel with the exclusion hard core constraint). The state space is $\Omega=\Omega_{1} \times \Omega_{2}$ with $\Omega_{i}=\mathbb{N}$, i.e. we have two sites each of which can accommodate an unlimited number of particles. For $\eta_{1}$ particles at site $1, \eta_{2}$ particles at site 2 , the rate of putting a particle from 1 to 2 is given by $2 \eta_{1}\left(2 \eta_{2}+1\right)$ and the rate of moving a particle from 2 to 1 is given by $2 \eta_{2}\left(2 \eta_{1}+1\right)$. We will see later how this process arises naturally as a dual of the Brownian Energy Process (BEP), see Sect. 5 below.

The matrix of the generator is given by

$$
\begin{aligned}
L\left(\eta_{1} \eta_{2}, \eta_{1}^{\prime} \eta_{2}^{\prime}\right)= & 2 \eta_{1}\left(2 \eta_{2}+1\right) \delta_{\eta_{1}^{\prime}, \eta_{1}-1} \delta_{\eta_{2}^{\prime}, \eta_{2}+1}+2 \eta_{2}\left(2 \eta_{1}+1\right) \delta_{\eta_{1}^{\prime}, \eta_{1}+1} \delta_{\eta_{2}^{\prime}, \eta_{2}-1} \\
& -\left(8 \eta_{1} \eta_{2}+2 \eta_{1}+2 \eta_{2}\right) \delta_{\eta_{1}, \eta_{1}^{\prime}} \delta_{\eta_{2}, \eta_{2}^{\prime}}
\end{aligned}
$$

The transposed of the generator can be written in terms of generators of a $S U(1,1)$ algebra as follows. On each site $i \in\{1,2\}$ we consider operators $K_{i}^{a}$ with $a \in\{+,-, 0\}$ given by

$$
\begin{aligned}
K_{i}^{+}\left|\eta_{i}\right\rangle & =\left(\eta_{i}+1 / 2\right)\left|\eta_{i}+1\right\rangle \\
K_{i}^{-}\left|\eta_{i}\right\rangle & =\eta_{i}\left|\eta_{i}-1\right\rangle \\
K_{i}^{0}\left|\eta_{i}\right\rangle & =\left(\eta_{i}+1 / 4\right)\left|\eta_{i}\right\rangle
\end{aligned}
$$


They satisfy the commutation relations of $S U(1,1)$ :

$$
\begin{aligned}
{\left[K_{i}^{0}, K_{i}^{ \pm}\right] } & = \pm K_{i}^{ \pm} \\
{\left[K_{i}^{-}, K_{i}^{+}\right] } & =2 K_{i}^{0}
\end{aligned}
$$

The transposed of the generator then reads

$$
L^{T}=4\left(K_{1}^{+} \otimes K_{2}^{-}+K_{1}^{-} \otimes K_{2}^{+}-2 K_{1}^{0} \otimes K_{2}^{0}+\frac{1}{8} \mathbb{1}_{1} \otimes \mathbb{1}_{2}\right)
$$

From the commutation relations, it is easy to see that $L^{T}$ commutes with $K^{a}=K_{1}^{a} \otimes \mathbb{1}_{2}+$ $\mathbb{1}_{1} \otimes K_{2}^{a}$, for $a \in\{+,-, 0\}$. A possible symmetry is then given by the matrix

$$
S=S_{1} \otimes S_{2}=e^{K_{1}^{+}} \otimes e^{K_{2}^{+}}
$$

which has matrix elements $S\left(\eta_{1} \eta_{2}, \xi_{1} \xi_{2}\right)=S_{1}\left(\eta_{1}, \xi_{1}\right) S_{2}\left(\eta_{2}, \xi_{2}\right)$ with

$$
S_{i}\left(\eta_{i}, \xi_{i}\right)=\left\langle\eta_{i}\left|e^{K_{1}^{+}}\right| \xi_{i}\right\rangle=\frac{\left(2 \eta_{i}-1\right) ! !}{\left(2 \xi_{i}-1\right) ! !\left(\eta_{i}-\xi_{i}\right) ! 2^{\eta_{i}-\xi_{i}}}
$$

A similarity transformation $L^{T}=Q^{-1} L Q$ to pass to the transposed is suggested ( $\mathrm{Re}$ mark 2.8) by the knowledge of the stationary measure of the dual-BEP (see Theorem 5.1)

$$
Q\left(\eta_{1} \eta_{2}, \eta_{1}^{\prime} \eta_{2}^{\prime}\right)=Q_{1}\left(\eta_{1}, \eta_{1}^{\prime}\right) Q_{2}\left(\eta_{2}, \eta_{2}^{\prime}\right)
$$

with

$$
Q_{i}\left(\eta_{i}, \xi_{i}\right)=\delta_{\eta_{i}, \xi_{i}}\left(\frac{\eta_{i} !}{\left(2 \eta_{i}-1\right) ! !} 2^{\eta_{i}}\right)^{-1}
$$

The self-duality function corresponding to $S$ of (29) and $Q$ of (30) then reads

$$
\begin{aligned}
D\left(\eta_{1} \eta_{2}, \xi_{1} \xi_{2}\right) & =D_{1}\left(\eta_{1}, \xi_{1}\right) D_{2}\left(\eta_{2}, \xi_{2}\right) \\
D_{i}\left(\eta_{i}, \xi_{i}\right) & =Q^{-1}\left(\eta_{i}, \eta_{i}\right) S_{i}\left(\eta_{i}, \xi_{i}\right)=2^{\xi_{i}} \frac{\eta_{i} !}{\left(\eta_{i}-\xi_{i}\right) !\left(2 \xi_{i}-1\right) ! !}
\end{aligned}
$$

\subsection{Self-duality for Independent Random Walkers}

This is a classical example which is included here for the sake of completeness (see also [13]). We have two site 1 and 2, and particles hop independently from 1 to 2 and from 2 to 1 at rate one. So the rate to put a particle from 1 to 2 in a configuration with $\eta_{1}$ particles at 1 and $\eta_{2}$ particles at 2 is simply $\eta_{1}$. The generator is given by the matrix

$$
L\left(\eta_{1} \eta_{2}, \eta_{1}^{\prime} \eta_{2}^{\prime}\right)=\eta_{2} \delta_{\eta_{1}, \eta_{1}^{\prime}-1} \delta_{\eta_{2}, \eta_{2}^{\prime}+1}+\eta_{1} \delta_{\eta_{1}, \eta_{1}^{\prime}+1} \delta_{\eta_{2}, \eta_{2}^{\prime}-1}+\left(-\eta_{1}-\eta_{2}\right) \delta_{\eta_{1}, \eta_{1}^{\prime}} \delta_{\eta_{2}, \eta_{2}^{\prime}}
$$

A self-duality function is $D=D_{1} \otimes D_{2}$ with

$$
D\left(\eta_{i}, \xi_{i}\right)=\frac{\eta_{i} !}{\left(\eta_{i}-\xi_{i}\right) !}
$$


The invariant measures are product of Poisson distributions and a possible conjugation is thus given by $Q=Q_{1} \otimes Q_{2}$ with

$$
Q_{i}\left(\eta_{i}, \xi_{i}\right)=\delta_{\eta_{i}, \xi_{i}} \frac{1}{\eta_{i} !}
$$

As a consequence, a symmetry of the generator is given by $S=S_{1} \otimes S_{2}$ with

$$
S_{i}\left(\eta_{i}, \xi_{i}\right)=\left(D_{i} Q_{i}\right)\left(\eta_{i}, \xi_{i}\right)=\frac{1}{\left(\eta_{i}-\xi_{i}\right) !}
$$

This symmetry comes once more from an underlying structure of creation and annihilation operators satisfying the Heisenberg algebra. Indeed, if one defines for $i \in\{1,2\}$ operators $a_{i}^{+}$and $a_{i}^{-}$which are represented on an Hilbert space with basis $|0\rangle,|1\rangle,|2\rangle, \ldots$ by operators working as

$$
\begin{aligned}
& a_{i}^{+}\left|\eta_{i}\right\rangle=\left|\eta_{i}+1\right\rangle \\
& a_{i}^{-}\left|\eta_{i}\right\rangle=\eta_{i}\left|\eta_{i}-1\right\rangle
\end{aligned}
$$

then one easily verifies the commutation relation

$$
\left[a_{i}^{-}, a_{i}^{+}\right]=\mathbb{1}_{i}
$$

In terms of these matrices, the transposed of the generator reads

$$
L^{T}=-\left(a_{1}^{+} \otimes \mathbb{1}_{2}-\mathbb{1}_{1} \otimes a_{2}^{+}\right)\left(a_{1}^{-} \otimes \mathbb{1}_{2}-\mathbb{1}_{1} \otimes a_{2}^{-}\right)
$$

which commutes with $a^{+}=a_{1}^{+} \otimes \mathbb{1}_{2}+\mathbb{1}_{1} \otimes a_{2}^{+}$. The symmetry $S$ in (32) is then recognized as $S=\exp \left(a_{1}^{+}\right) \otimes \exp \left(a_{2}^{+}\right)$.

\subsection{Duality between Independent Random Walkers and a Deterministic System}

As an example of application of Theorem 2.10 we consider again a system of independent random walkers jumping between sites 1 and 2 . We show that this system is dual to a deterministic system evolving according to ordinary differential equations.

We consider the "abstract" operator $\mathcal{L}$

$$
\mathcal{L}=-\left(a_{1}^{+}-a_{2}^{+}\right)\left(a_{1}^{-}-a_{2}^{-}\right)
$$

where $a_{i}^{+}, a_{j}^{-}$are operators satisfying the canonical commutation relations

$$
\left[a_{i}^{-}, a_{j}^{+}\right]=\delta_{i, j} \mathbb{1}
$$

One way to represent the previous operator is by considering

$$
a_{i}^{-}=\frac{\partial}{\partial x_{i}}, \quad a_{i}^{+}=x_{i}, \quad i \in\{1,2\}
$$

which obviously satisfy (36). In this case the operator (35) takes the form

$$
L=-\left(x_{1}-x_{2}\right)\left(\frac{\partial}{\partial x_{1}}-\frac{\partial}{\partial x_{2}}\right)
$$


which is the generator of the deterministic system of differential equations

$$
\begin{aligned}
& \frac{d x_{1}(t)}{d t}=-\left(x_{1}(t)-x_{2}(t)\right) \\
& \frac{d x_{2}(t)}{d t}=\left(x_{1}(t)-x_{2}(t)\right)
\end{aligned}
$$

with solutions

$$
\begin{aligned}
& x_{1}(t)=\frac{x_{1}(0)+x_{2}(0)}{2}+\frac{x_{1}(0)-x_{2}(0)}{2} e^{-2 t} \\
& x_{2}(t)=\frac{x_{1}(0)+x_{2}(0)}{2}-\frac{x_{1}(0)-x_{2}(0)}{2} e^{-2 t}
\end{aligned}
$$

Another possible way to represent the operator (35) has just been seen in the previous paragraph. In this case the creation and annihilation operators are represented as matrices with elements given by (33) and then the operator (35) can be seen as the transposed of the generator for a system on independent random walkers

$$
L_{\text {dual }}^{T}=-\left(a_{1}^{+} \otimes \mathbb{1}_{2}-\mathbb{1}_{1} \otimes a_{2}^{+}\right) \circ\left(a_{1}^{-} \otimes \mathbb{1}_{2}-\mathbb{1}_{1} \otimes a_{2}^{-}\right)
$$

It is immediately checked that the function

$$
D(x, \xi)=D\left(x_{1}, \xi_{1}\right) D\left(x_{2}, \xi_{2}\right)
$$

with

$$
D\left(x_{i}, \xi_{i}\right)=x_{i}^{\xi_{i}}
$$

gives a conjugation between $L$ and $L_{\text {dual }}^{T}$, namely

$$
L D(x, \xi)=D L_{d u a l}^{T}(x, \xi)
$$

In this case, this relation reads more explicitely,

$$
\begin{aligned}
& -\left(x_{1}-x_{2}\right)\left(\frac{\partial}{\partial x_{1}}-\frac{\partial}{\partial x_{2}}\right)\left(x_{1}^{n_{1}} x_{2}^{n_{2}}\right) \\
& =n_{1} x_{1}^{n_{1}-1} x_{2}^{n_{2}+1}+n_{2} x_{1}^{n_{1}+1} x_{2}^{n_{2}-1}-\left(n_{1}+n_{2}\right) x_{1}^{n_{1}} x_{2}^{n_{2}}
\end{aligned}
$$

This implies that $x(t)$ and $\xi(t)$ are each other dual with duality function $D$ and the following relation holds

$$
x_{1}(t)^{\xi_{1}} x_{2}(t)^{\xi_{2}}=\mathbb{E}_{\xi_{1}, \xi_{2}}\left(x_{1}(0)^{\xi_{1}(t)} x_{2}(0)^{\xi_{2}(t)}\right)
$$

where the expectation in the r.h.s. is over the independent random walkers starting from initial configuration with $\eta_{1}$ particles at 1 and $\eta_{2}$ particles at 2 . We will come back to this example in Sect. 6.4.

Remark 3.1 In the last example, we can still use other representations of the operators $a_{i}^{-}, a_{i}^{+}$, satisfying the commutation relation $\left[a_{i}^{-}, a_{j}^{+}\right]=\delta_{i j}$, such that the abstract operator 
(35) is the generator of a Markovian diffusion process. E.g., if we choose

$$
\begin{aligned}
& a_{i}^{+}=-\frac{\partial}{\partial x_{i}}+x_{i} \\
& a_{i}^{-}=\frac{\partial}{\partial x_{i}}
\end{aligned}
$$

then the abstract operator (35) reads

$$
\mathcal{L}=\left(\frac{\partial}{\partial x_{1}}-\frac{\partial}{\partial x_{2}}\right)^{2}+\left(x_{1}-x_{2}\right)\left(\frac{\partial}{\partial x_{1}}-\frac{\partial}{\partial x_{2}}\right)
$$

which is the generator of the (degenerate) diffusion: the "coordinate $\left(x_{1}-x_{2}\right) / 2$ undergoes a Brownian motion and $\left(x_{1}+x_{2}\right) / 2$ remains constant. So in that case we also have duality

$$
\mathbb{E}_{x_{1}, x_{2}}\left(D\left(x_{1}(t), n_{1}\right) D\left(x_{2}(t), n_{2}\right)\right)=\mathbb{E}_{n_{1}, n_{2}}\left(D\left(x_{1}, n_{1}(t)\right) D\left(x_{2}, n_{2}(t)\right)\right)
$$

where $D(x, n)$ can be found by the recursion

$$
D(x, n+1)=a^{+} D(x, n)
$$

e.g. the first five polynomials are

$$
\begin{aligned}
& D(x, 0)=1, \quad D(x, 1)=x, \quad D(x, 2)=x^{2}-1 \\
& D(x, 3)=-3 x+x^{3}, \quad D(x, 4)=3-6 x^{2}+x^{4}
\end{aligned}
$$

\section{Symmetric Exclusion Processes}

In this section we study the $2 j$-SEP (with $j \in \mathbb{N} / 2$ ), i.e. exclusion processes with at most $2 j$ particles per site, on a graph $S$. We show how we can understand self-duality for the $2 j$-SEP from "classical duality" (in the sense of [9]) of the symmetric exclusion process on special graphs. We also consider two limits $j \rightarrow \infty$ leading to a deterministic process or a system of independent random walkers. Finally, we consider the boundary driven case, and show that we have a dual with absorbing boundaries.

\subsection{Symmetric Exclusion on Ladder Graphs}

Consider a countable set $\mathcal{S}$, to be thought of as the underlying lattice, and a finite set $\mathcal{I}$ with cardinality $2 j \in \mathbb{N}$. The set $\mathcal{I}$ is to be thought of as a "ladder" on each site with $2 j$ levels.

The state space of SEP on the ladder graph $\mathcal{S} \times \mathcal{I}$ is $\Omega=\{0,1\}^{\mathcal{S} \times \mathcal{I}}$. A configuration $\zeta \in \Omega$ is called finite if it contains a finite number of particles, i.e., if $\sum_{i \in \mathcal{S}, \alpha \in \mathcal{I}} \zeta(i, \alpha)<\infty$. The process is described as follows. Let $p(i, l)$ denote a symmetric random walk kernel on $\mathcal{S}$, i.e., $p(i, l)=p(l, i) \geq 0, \sum_{l \in \mathcal{S}} p(i, l)=1$. At each site $i \in \mathcal{S}$ and level $\alpha \in \mathcal{I}$, there is at most one particle. Each particle attempts to jump at rate $p(i, l)$ to a site $l \in \mathcal{S}$ and level $\beta \in \mathcal{I}$.

More formally, the SEP on a ladder graph $\mathcal{S} \times \mathcal{I}$ is the process with the following generator on local functions $f: \Omega \rightarrow \mathbb{R}$

$$
L f(\zeta)=\sum_{i, l \in \mathcal{S}} \sum_{\alpha, \beta \in \mathcal{I}} p(i, l)\left(\zeta(i, \alpha)(1-\zeta(l, \beta))\left(f\left(\zeta^{(i, \alpha),(l, \beta)}\right)-f(\zeta)\right)\right.
$$


where $\zeta^{(i, \alpha),(l, \beta)}$ denotes the configuration obtained from $\eta$ by removing a particle at site $i$ level $\alpha$ and placing it at site $l$ level $\beta$. Since this process is a symmetric exclusion process on a special graph, then it is self-dual in the following sense:

Proposition 4.1 Define for $\zeta, \tilde{\zeta} \in \Omega, \tilde{\zeta}$ finite,

$$
D(\zeta, \tilde{\zeta})=\prod_{i, \alpha: \tilde{\zeta}(i, \alpha)=1} \zeta(i, \alpha)
$$

then we have the duality relation from [9]

$$
\mathbb{E}_{\zeta} D\left(\zeta_{t}, \tilde{\zeta}\right)=\mathbb{E}_{\tilde{\zeta}} D\left(\zeta, \tilde{\zeta}_{t}\right)
$$

where $\zeta_{t}, \tilde{\zeta}_{t}$ are independent copies of the ladder SEP with generator (42) starting from $\zeta$, $\operatorname{resp} . \tilde{\zeta}$.

\subsection{From the Ladder SEP to the $2 j$-SEP}

To define the $2 j$-SEP on a graph $\mathcal{S}$ we consider, for a given SEP on a ladder graph $\mathcal{S} \times \mathcal{I}$ with $2 j$ levels, the process which consists of giving at time $t>0$, and each site $i \in \mathcal{S}$ the number of levels $(i, \alpha)$ which are occupied in $\zeta_{t}$. More precisely, define the map $\pi: \Omega \rightarrow$ $\Omega^{(j)}=\{0,1, \ldots, 2 j\}^{\mathcal{S}}$

$$
\zeta \mapsto \pi(\zeta)=\eta \quad \text { with } \eta_{i}=\sum_{\alpha \in \mathcal{I}} \zeta(i, \alpha)
$$

Then we have the following theorem.

Theorem 4.2 Let $\zeta_{t}$ be the ladder sep with generator (42). Then the following holds:

(a) $\eta_{t}=\pi\left(\zeta_{t}\right)$ is a Markov process on $\Omega^{(j)}$ with generator

$$
L^{(j)} f(\eta)=\sum_{i, l \in S} p(i, l) \eta_{i}\left(2 j-\eta_{l}\right)\left(f\left(\eta^{i, l}\right)-f(\eta)\right)
$$

This process will be called the $2 j$-SEP or reduced ladder SEP with $2 j$ levels.

(b) The process $\eta_{t}$ with generator $L^{(j)}$ is self-dual with duality function

$$
D(\eta, \xi)=\prod_{i \in S} \frac{\left(\begin{array}{l}
\eta_{i} \\
\xi_{i}
\end{array}\right)}{\left(\begin{array}{c}
2 j \\
\xi_{i}
\end{array}\right)}
$$

for $\xi \leq \eta$ a configuration with a finite number of particles ( $D$ is defined to be zero in other cases). More precisely, we have

$$
\mathbb{E}_{\eta} D\left(\eta_{t}, \xi\right)=\mathbb{E}_{\xi} D\left(\eta, \xi_{t}\right)
$$

(c) The extremal invariant measures are of the form

$$
v_{\rho}^{(j)}=\bigotimes_{i \in S} \operatorname{Bin}\left(2 j, \rho_{i}\right)
$$


where $\rho_{i}$ is harmonic for $p(i, l)$, i.e., such that

$$
\sum_{l} p(i, l) \rho_{l}=\rho_{i}
$$

In particular, if the only bounded harmonic functions are constants, then the only extremal invariant measures are products of binomials with constant density.

Proof For point (a) remark that the jump rates in the generator (45) only depend on the number of particles at a site, and not on the levels. Therefore, if $f: \Omega \rightarrow \mathbb{R}$ depends on $\zeta$ only through $\eta=\pi(\zeta)$, i.e., if $f(\zeta)=\psi(\pi(\zeta))=\psi(\eta)$, then

$$
L f(\zeta)=L^{(j)} \psi(\eta)
$$

Therefore, for every local function $\psi: \Omega^{(j)} \rightarrow \mathbb{R}$,

$$
\psi\left(\pi\left(\zeta_{t}\right)\right)-\psi\left(\pi\left(\zeta_{0}\right)\right)-\int_{0}^{t} L^{(j)}(\psi)\left(\pi\left(\zeta_{s}\right)\right) d s=M_{t}
$$

is a martingale w.r.t. the filtration $\mathcal{F}_{t}=\sigma\left\{\pi(\zeta)_{s}: 0 \leq s \leq t\right\}$. This shows that $\pi\left(\zeta_{t}\right)$ is a solution of the martingale problem associated to the generator $L^{(j)}$, and hence coincides with the unique Markov process generated by $L^{(j)}$ (see Theorem 4.1, p. 182, of [4]).

Now we turn to point (b). At each site $i \in S$ we choose $\xi_{i}$ levels at random. For a given configuration $\eta \in \Omega_{j}$, we choose $\zeta \in \Omega$ consistent, i.e., such that $\pi(\zeta)=\eta$. Then the probability (w.r.t. to the random choices) that all chosen levels are occupied in $\zeta$ is exactly equal to $D(\eta, \xi)$. As $\pi\left(\zeta_{t}\right)=\eta_{t}$, the probability that the chosen levels are occupied at time $t$ (i.e., in $\zeta_{t}$ ) is given by $\mathbb{E}_{\eta} D\left(\eta_{t}, \xi\right)$. By self-duality of the ladder SEP (Proposition 4.1), the event that at time $t>0$ the chosen levels are occupied is the same as the probability that the particles evolving from the chosen levels during a time $t$ find themselves at positions which are occupied in $\zeta$. The latter probability equals $\mathbb{E}_{\xi} D\left(\xi_{t}, \eta\right)$.

Point (c) follows from the fact that for the ladder SEP with generator (42), the product Bernoulli measures indexed by harmonic functions of $p(i, j)$ are the extremal invariant measures (see [9] for details) and the image measure of a product of Bernoulli measure under $\pi$ is a product of Binomial measures.

\subsection{Limiting Processes as $j \rightarrow \infty$}

In this section we show that for large $j$ the $2 j$-SEP converges, when considered on an appropriate time scale, either to a system of independent random walkers or to a deterministic limit, depending on the initial density (it was already recognized in [13] that the limit $j \rightarrow \infty$ leads to non-interacting particles, here we give a precise statement). We remind the reader that for independent random walkers on a graph $\mathcal{S}$, the configuration space is $\Omega_{\infty}=\mathbb{N}^{\mathcal{S}}$ and the generator is given by

$$
L_{\text {irw }} f(\eta)=\sum_{i, l \in \mathcal{S}} \eta(i) p(i, l)\left(f\left(\eta^{i, l}\right)-f(\eta)\right)
$$

The stationary measures are products of Poisson measures, and the process with generator (50) is self-dual with self-duality function

$$
D_{i r w}(\eta, \xi)=\prod_{i \in S} \frac{\eta_{i} !}{\left(\eta_{i}-\xi_{i}\right) !}
$$

for finite configurations $\xi \leq \eta$, and $D=0$ otherwise. 
The relation with the reduced ladder SEP for large $j$ is given in the following theorem.

Theorem 4.3 Consider the process $\left\{\eta_{t}^{(j)}: t \geq 0\right\}$ with generator (45) started from initial configuration $\eta^{(j)} \in \Omega^{(j)}$. Suppose that, as $j \rightarrow \infty, \eta^{(j)} \rightarrow \eta \in \Omega_{\infty}$, then the process $\left\{\eta_{t / 2 j}^{(j)}\right.$ : $t \geq 0\}$ converges weakly in path space to a system of independent random walkers with generator (50) and initial configuration $\eta$.

Proof The process $\left\{\eta_{t / 2 j}^{(j)}: t \geq 0\right\}$ has generator

$$
\mathcal{L}_{j}^{\prime}=\frac{1}{2 j} L^{(j)}
$$

In order to have a sequence of processes all defined on the same sample space $\Omega^{(\infty)}$ we consider the auxiliary process on $\mathbb{N}^{S}$ with generator

$$
\mathcal{L}_{j}^{\prime \prime} f(\eta)=\sum_{i, l \in S} p(i, l) \eta_{i}\left(1-\frac{\eta_{l}}{2 j}\right) I\left(\eta_{i} \leq 2 j\right) I\left(\eta_{l} \leq 2 j\right)\left(f\left(\eta^{i, l}\right)-f(\eta)\right)
$$

This auxiliary process behaves as the process with generator $\mathcal{L}_{j}^{\prime}$ except for sites which have more than $2 j$ particles, which are frozen. Started from an initial configuration with all sites having at most $2 j$ particles, this process coincides with the process $\eta_{t / 2 j}$. For any local function $f: \mathbb{N}^{S} \rightarrow \mathbb{R}$, we then have

$$
\begin{aligned}
\lim _{j \rightarrow \infty} \mathcal{L}_{j}^{\prime \prime} f(\eta) & =\lim _{j \rightarrow \infty} \sum_{i, l \in S} p(i, l) \eta_{i}\left(1-\frac{\eta_{l}}{2 j}\right) I\left(\eta_{i} \leq 2 j\right) I\left(\eta_{l} \leq 2 j\right)\left(f\left(\eta^{i, l}\right)-f(\eta)\right) \\
& =\mathcal{L}_{\text {irw }} f(\eta)
\end{aligned}
$$

Therefore, by the Trotter-Kurtz theorem (see Theorem 2.5 of [4]), this implies that the corresponding processes $\eta_{t / 2 j}$ converge weakly on path space as $j \rightarrow \infty$ to the process with generator $\mathcal{L}_{\text {irw }}$.

To see that (51) is (up to a multiplicative consant) a limit of duality functions of the $2 j$-SEP, we start from the symmetry (22) and use the similarity transformation with $Q^{(j)}$ given by

$$
Q^{(j)}(\eta, \xi)=\prod_{i \in \mathcal{S}} Q_{i}^{(j)}\left(\eta_{i}, \xi_{i}\right)
$$

with

$$
Q_{i}^{(j)}\left(\eta_{i}, \xi_{i}\right)=\delta_{\eta_{i}, \xi_{i}}\left(\begin{array}{c}
2 j \\
\eta_{i}
\end{array}\right)\left(\frac{1}{2 j}\right)^{\eta_{i}}\left(1-\frac{1}{2 j}\right)^{2 j-\eta_{i}}
$$

Then the duality functions

$$
\tilde{D}^{(j)}=Q^{(j)^{-1}} S
$$

with $S$ defined in (32), satisfy

$$
\lim _{j \rightarrow \infty} \tilde{D}^{(j)}=e D_{i r w}
$$


Another possible limit is obtained when the initial condition has a number of particles that diverges proportional to $j$. This limit, as can be understood from the law of large numbers, is deterministic.

Theorem 4.4 Consider the process

$$
x_{i}^{(j)}(t)=\frac{\eta_{t / 2 j}^{(j)}}{2 j}
$$

and suppose that $x_{i}^{(j)}(0) \rightarrow x_{i}(0) \in[0,1]$ for all $i \in \mathcal{S}$ as $j \rightarrow \infty$. Then we have that $x_{i}^{(j)}(t)$ converges to a deterministic system of coupled differential equations with generator

$$
L f(x)=\sum_{i, l \in S} p(i, l) x_{i}\left(1-x_{l}\right)\left(\frac{\partial}{\partial x_{l}}-\frac{\partial}{\partial x_{i}}\right)
$$

Proof The generator of the process $x_{i}^{(j)}(t)$ reads

$$
\mathcal{L}_{j}^{\prime} f(x)=2 j \sum_{i, l} p(i, l) x_{i}\left(1-x_{l}\right)\left(f\left(x^{(j) ; i, l}\right)-f\left(x^{(j)}\right)\right)
$$

where $x^{(j), i, l}$ arises from $x^{(j)}$ by removing a unit $1 / 2 j$ from $i \in \mathcal{S}$ and putting it at $l \in \mathcal{S}$. Therefore, for a local smooth function $f:[0,1]^{S} \rightarrow \mathbb{R}$ we have, by Taylor expansion

$$
\mathcal{L}_{j}^{\prime} f(x)=\sum_{i, l} p(i, l) x_{i}\left(1-x_{l}\right)\left(\frac{\partial}{\partial x_{l}}-\frac{\partial}{\partial x_{i}}\right) f(x)+O\left(\frac{1}{2 j}\right)
$$

The result then follows once more from the Trotter Kurtz theorem. Since the limiting generator is a first order differential operator, the corresponding process is deterministic.

\subsection{Boundary Driven Case}

We first discuss a duality theorem for standard (i.e. $j=1 / 2$ ) symmetric exclusion with extra creation and annihilation of particles at the boundary (duality for open boundaries was also exploited in $[15,16])$. The context is a countable set $\mathcal{S}$, of which we distinguish a subset $\partial \mathcal{S} \subset \mathcal{S}$ called the boundary. We then consider the generator

$$
\begin{aligned}
\mathcal{L} f(\eta)= & \sum_{i, l \in \mathcal{S}} p(i, l)\left(\eta(i)(1-\eta(l))\left(f\left(\eta^{i, l}\right)-f(\eta)\right)\right. \\
& +\sum_{i \in \partial \mathcal{S}}\left(1-\rho_{i}\right) \eta(i)\left(f\left(\eta^{i}\right)-f(\eta)\right)+\rho_{i}(1-\eta(i))\left(f\left(\eta^{i}\right)-f(\eta)\right)
\end{aligned}
$$

where $0<\rho_{i}<1$ represent the densities of the particle reservoirs with which the system is in contact at the boundary sites, and where $\eta^{i}$ is the configuration obtained from $\eta$ by flipping the occupancy at $i$.

The first part of the generator represents the hopping of particles on $\mathcal{S}$ according to a symmetric exclusion process, whereas the second part represents creation and annihilation of particles at the boundary sites.

To introduce duality for this process, we introduce a set $\partial_{e} \mathcal{S}$ of sink sites, and a bijection $i \mapsto i_{e}$ which associates each site $i \in \partial \mathcal{S}$ to a sink site. The dual process will then be 
a process that behaves as the exclusion process in the bulk, but particles can leave the system via boundary sites to sink sites, and will then be stuck at sink sites. More precisely, a configuration of the dual process is a map

$$
\xi: \mathcal{S} \cup \partial_{e} \mathcal{S} \rightarrow \mathbb{N}
$$

such that $\xi(i) \in\{0,1\}$ for $i \in \mathcal{S}$ (only the sink sites can contain more than one particle). We call $\Omega_{\text {dual }}$ the set of all configurations of the dual process.

The duality function is defined as follows: for $\eta \in\{0,1\}^{\mathcal{S}}, \xi \in \Omega_{\text {dual }}$

$$
D(\eta, \xi)=\left(\prod_{i \in \mathcal{S}: \xi(i)=1} \eta(i)\right)\left(\prod_{i \in \partial_{e} \mathcal{S}} \rho_{i}^{\xi_{i}}\right)
$$

The idea here is that we have the ordinary duality function for the sites $i \in \mathcal{S}$ and for the sink sites, we replace the variable $\eta_{i}$ by its expectation $\rho_{i}$, corresponding to the stationary measure of the boundary generator $L_{i}$.

The generator of the dual process is then defined as follows:

$$
\mathcal{L}_{\text {dual }} f(\xi)=\sum_{i, l \in \mathcal{S}} p(i, l)\left(\xi(i)(1-\xi(l))\left(f\left(\xi^{i, l}\right)-f(\xi)\right)+\sum_{i \in \partial \mathcal{S}} \xi(i)\left(f\left(\xi^{i, i_{e}}\right)-f(\xi)\right)\right.
$$

We then have

Theorem 4.5 The boundary driven exclusion process $\left(\eta_{t}\right)_{t \geq 0}$ with generator $\mathcal{L}$ in (56) and the process $\left(\xi_{t}\right)_{t \geq 0}$ with generator $\mathcal{L}_{\text {dual }}$ in (58) are dual with duality function $D(\eta, \xi)$ given by (57), i.e.,

$$
\mathbb{E}_{\eta} D\left(\eta_{t}, \xi\right)=\mathbb{E}_{\xi} D\left(\eta, \xi_{t}\right)
$$

Proof Abbreviate

$$
L_{i} f(\eta)=\left(1-\rho_{i}\right) \eta(i)\left(f\left(\eta^{i}\right)-f(\eta)\right)+\rho_{i}(1-\eta(i))\left(f\left(\eta^{i}\right)-f(\eta)\right)
$$

and

$$
L_{i}^{\text {dual }} f(\xi)=\xi(i)\left(f\left(\xi^{i, i_{e}}\right)-f(\xi)\right)
$$

For $f(\eta)=\eta(i)$ one sees that

$$
L_{i} f(\eta)=\rho_{i}-\eta(i)
$$

and hence for $\xi$ a dual configuration which is non-zero only on the sites $i \in \partial \mathcal{S}$ and on the corresponding sink site $i_{e} \in \partial_{e} \mathcal{S}$, we find

$$
\begin{aligned}
L_{i} D(\eta, \xi) & =\rho_{i}^{\xi_{i e}}\left(L_{i}(\eta(i) \xi(i)+(1-\xi(i)))\right) \\
& =\xi(i) \rho_{i}^{\xi_{i e}}\left(\rho_{i}-\eta(i)\right) \\
& =\xi(i)\left(\rho_{i}^{\xi_{i e}+1}-\rho_{i}^{\xi_{i e}} \eta(i)\right)=L_{i}^{\text {dual }} D(\xi, \eta)
\end{aligned}
$$

From that and the self-duality of the symmetric exclusion process, it follows that

$$
\mathcal{L} D(\eta, \xi)=\mathcal{L}_{\text {dual }} D(\eta, \xi)
$$


In order to apply this duality result for the boundary driven process with generator (45), we first look at the boundary driven exclusion process on a ladder graph. More precisely, for $\zeta \in\{0,1\}^{\mathcal{S} \times \mathcal{I}}$ we consider the process $\left(\zeta_{t}\right)_{t \geq 0}$ with generator

$$
\begin{aligned}
\mathcal{L} f(\zeta)= & \sum_{i, l \in \mathcal{S}} \sum_{\alpha, \beta \in \mathcal{I}} p(i, l)\left(\zeta(i, \alpha)(1-\zeta(l, \beta))\left(f\left(\zeta^{(i, \alpha),(l, \beta)}\right)-f(\zeta)\right)\right. \\
& +\sum_{i \in \partial \mathcal{S}} \sum_{\alpha \in \mathcal{I}}\left(1-\rho_{i}\right) \zeta(i, \alpha)\left(f\left(\zeta^{(i, \alpha)}\right)-f(\zeta)\right) \\
& +\rho_{i}(1-\zeta(i, \alpha))\left(f\left(\zeta^{(i, \alpha)}\right)-f(\zeta)\right)
\end{aligned}
$$

In words, this process is the ladder SEP, with additional boundary driving, where the creation and annihilation rate of particles at the boundary sites does not depend on the level. If we consider the reduced process, consisting of counting at each site $i \in \mathcal{S}$ how many levels in $\mathcal{I}$ are occupied, i.e. $\eta_{t}=\pi\left(\zeta_{t}\right)$ then we recover once again a Markov process (cfr. Theorem 4.2). This process, defined on the state space $\Omega^{(j)}=\{0,1, \ldots, 2 j\}^{\mathcal{S}}$ and called the boundary driven $2 j$-SEP, has generator

$$
\begin{aligned}
\mathcal{L}_{j} f(\eta)= & \sum_{i, l \in \mathcal{S}} p(i, l)\left(\eta(i)(2 j-\eta(l))\left(f\left(\eta^{i, l}\right)-f(\eta)\right)\right. \\
& +\sum_{i \in \partial \mathcal{S}}\left(1-\rho_{i}\right) \eta(i)\left(f\left(\eta^{(i, \alpha)}\right)-f(\eta)\right)+\rho_{i}\left(2 j-\eta_{i}\right)\left(f\left(\eta^{i}\right)-f(\eta)\right)
\end{aligned}
$$

It turns out that the boundary driven $2 j$-SEP has a nice dual too. To introduce this dual, we consider admissible dual configurations as maps $\xi: \mathcal{S} \cup \partial_{e} \mathcal{S} \rightarrow \mathbb{N}$ such that $0 \leq \xi(i) \leq 2 j$ for $i \in \mathcal{S}$ (only the sink sites can contain more that $2 j$ particles). The generator of the dual of the boundary driven $2 j$-SEP is a process on admissible dual configurations, defined by

$$
\begin{aligned}
\mathcal{L}_{j}^{\text {dual }} f(\xi)= & \sum_{i, l \in \mathcal{S}} p(i, l)\left(\xi(i)(2 j-\xi(l))\left(f\left(\xi^{i, l}\right)-f(\xi)\right)\right. \\
& +\sum_{i \in \partial \mathcal{S}} \xi(i)\left(f\left(\xi^{i, i_{e}}\right)-f(\xi)\right)
\end{aligned}
$$

From Theorem 4.5 we then infer, in the same way as we derived Theorem 4.2 the following.

Theorem 4.6 Let $\left(\eta_{t}\right)_{t \geq 0}$ denote the boundary driven $2 j$-SEP with generator (65). Then $\left(\eta_{t}\right)_{t \geq 0}$ is dual to the process $\left(\xi_{t}\right)_{t \geq 0}$ with generator (66) with duality function given by

$$
D(\eta, \xi)=\prod_{i \in \partial_{e} \mathcal{S}} \rho_{i}^{\xi_{i}} \prod_{i \in \mathcal{S}} \frac{\left(\begin{array}{l}
\eta_{i} \\
\xi_{i}
\end{array}\right)}{\left(\begin{array}{l}
2 j \\
\xi_{i}
\end{array}\right)}
$$

Proof Denote for $i \in \partial \mathcal{S}$,

$$
\mathcal{L}_{i} f(\eta)=\left(1-\rho_{i}\right) \eta(i)\left(f\left(\eta^{(i, \alpha)}\right)-f(\eta)\right)+\rho_{i}(2 j-\eta(i))\left(f\left(\eta^{i}\right)-f(\eta)\right)
$$

and

$$
\mathcal{L}_{i}^{\text {dual }} f(\xi)=\xi(i)\left(f\left(\xi^{i, i_{e}}\right)-f(\xi)\right)
$$


One then easily computes that for $\xi$ a dual configuration which is non-zero only on the sites $i \in \partial \mathcal{S}$ and on the corresponding sink site $i_{e} \in \partial_{e} \mathcal{S}$,

$$
\begin{aligned}
\mathcal{L}_{i} D(\eta, \xi) & =\rho_{i}^{\xi_{i_{e}}} \frac{1}{\left(\begin{array}{c}
2 j \\
\xi_{i}
\end{array}\right)} \mathcal{L}_{i}\left(\left(\begin{array}{c}
\eta_{i} \\
\xi_{i}
\end{array}\right)\right) \\
& =\rho_{i}^{\xi_{i}} \frac{1}{\left(\begin{array}{c}
2 j \\
\xi_{i}
\end{array}\right)}\left[\left(1-\rho_{i}\right) \eta_{i}\left(\left(\begin{array}{c}
\eta_{i}-1 \\
\xi_{i}
\end{array}\right)-\left(\begin{array}{c}
\eta_{i} \\
\xi_{i}
\end{array}\right)\right)+\left(2 j-\eta_{i}\right) \rho_{i}\left(\left(\begin{array}{c}
\eta_{i}+1 \\
\xi_{i}
\end{array}\right)-\left(\begin{array}{c}
\eta_{i} \\
\xi_{i}
\end{array}\right)\right)\right] \\
& =\xi_{i}\left(\rho_{i}^{\xi_{i e}+1}-\rho_{i}\right) \frac{\left(\begin{array}{c}
\eta_{i} \\
\xi_{i}
\end{array}\right)}{\left(\begin{array}{c}
2 j \\
\xi_{i}
\end{array}\right)}=\mathcal{L}_{i}^{\text {dual }} D(\eta, \xi)
\end{aligned}
$$

The result then follows from combination of this fact and the duality relation (47).

\section{The Brownian Momentum Process and $S U(1,1)$ Symmetry}

In this section we study the Brownian momentum process that was introduced in $[2,5]$. We will recover duality [6] in the context of our main Theorems and study the reversible measures of the dual process.

\subsection{Generator and Quantum Spin Chain}

Let $\mathcal{S}$ be a countable set and $p(i, j)$ a symmetric random walk transition probability on $\mathcal{S}$. The Brownian momentum process is a Markov process $\left(x_{t}\right)_{t \geq 0}$ on $\mathbb{R}^{\mathcal{S}}$, with generator

$$
L=\sum_{i, j \in \mathcal{S}} p(i, j) L_{i j}
$$

where

$$
L_{i j}=\left(x_{i} \frac{\partial}{\partial x_{j}}-x_{j} \frac{\partial}{\partial x_{i}}\right)^{2}
$$

and $p(i, j)$ is a symmetric random walk kernel on $\mathcal{S}$.

The generator $L_{i j}$ conserves the energy $x_{i}^{2}+x_{j}^{2}$ and generates a Brownian rotation of the angle $\theta_{i j}=\arctan \left(x_{j} / x_{i}\right)$. The interpretation of the generator (68) is then as follows: each bond independently, at rate $p(i, j)$ undergoes a Brownian rotation of its angle $\theta_{i j}=$ $\arctan \left(x_{j} / x_{i}\right)$. An important example to keep in mind is $\mathcal{S}=\mathbb{Z}^{d}$, and $p(i, j)$ the nearest neighbor symmetric random walk.

The processes with generator $L$ can be related to quantum spin chains [6]. Consider the operators

$$
\begin{aligned}
K_{i}^{+} & =\frac{1}{2} x_{i}^{2} \\
K_{i}^{-} & =\frac{1}{2} \frac{\partial^{2}}{\partial x_{i}^{2}} \\
K_{i}^{0} & =\frac{1}{4}\left(\frac{\partial}{\partial x_{i}}\left(x_{i} \cdot\right)+x_{i} \frac{\partial}{\partial x_{i}}\right)
\end{aligned}
$$


which satisfy the commutation relations of $S U(1,1)$ :

$$
\begin{aligned}
{\left[K_{i}^{0}, K_{i}^{ \pm}\right] } & = \pm K_{i}^{ \pm} \\
{\left[K_{i}^{-}, K_{i}^{+}\right] } & =2 K_{i}^{0}
\end{aligned}
$$

Then the negative of the adjoint of the generator $L$ can be seen as the quantum "Hamiltonian"

$$
H=-L^{*}=-4 \sum_{i j \in \mathcal{S}} p(i, j)\left(K_{i}^{+} K_{j}^{-}+K_{i}^{-} K_{j}^{+}-2 K_{i}^{0} K_{j}^{0}+\frac{1}{8}\right)
$$

with spin satisfying the $S U(1,1)$ algebra (in a representation with spin value $1 / 4$ ).

\subsection{Dual Process}

In [6] we showed that the process with generator $L$ in (68) and (69) has a dual, which is a system of interacting random walkers on $\mathcal{S}$. We show here how this dual process comes out of the structure of the Hamiltonian (72).

We notice that the $S U(1,1)$ group admits a discrete (infinite dimensional) representation as (unbounded) operators on $l_{2}(\mathbb{N})$ :

$$
\begin{aligned}
\mathcal{K}_{i}^{+}\left|\xi_{i}\right\rangle & =\left(\frac{1}{2}+\xi_{i}\right)\left|\xi_{i}+1\right\rangle \\
\mathcal{K}_{i}^{-}\left|\xi_{i}\right\rangle & =\xi_{i}\left|\xi_{i}-1\right\rangle \\
\mathcal{K}_{i}^{0}\left|\xi_{i}\right\rangle & =\left(\xi_{i}+\frac{1}{4}\right)\left|\xi_{i}\right\rangle
\end{aligned}
$$

where $i \in \mathcal{S}$ and $\xi_{i} \in \mathbb{N}$ and $|0\rangle,|1\rangle,|2\rangle, \ldots$ denotes the canonical basis on $l_{2}(\mathbb{N})$. It is immediately checked that the (unbounded) operators in (73) satisfy the $S U(1,1)$ commutation relations in (71). We then define a new generator via the same Hamiltonian as in (72), but now in the representation (73):

$$
H_{\text {dual }}=-L_{\text {dual }}^{*}=-4 \sum_{i j \in \mathcal{S}} p(i, j)\left(\mathcal{K}_{i}^{+} \mathcal{K}_{j}^{-}+\mathcal{K}_{i}^{-} \mathcal{K}_{j}^{+}-2 \mathcal{K}_{i}^{0} \mathcal{K}_{j}^{0}+\frac{1}{8}\right)
$$

From the previous equation and using the representation (73) we deduce that the Hamiltonian above defines a Markov process $\left(\xi_{t}\right)_{t \geq 0}$ with state space $\mathbb{N}^{\mathcal{S}}$ and generator

$$
L_{\text {dual }}=\sum_{i, j \in \mathcal{S}} p(i, j) L_{i j}^{\text {dual }}
$$

where

$$
\begin{aligned}
L_{i j}^{\text {dual }} f(\xi)= & 2 \xi_{i}\left(2 \xi_{j}+1\right)\left(f\left(\xi^{i, j}\right)-f(\xi)\right) \\
& +2 \xi_{j}\left(2 \xi_{i}+1\right)\left(f\left(\xi^{j, i}\right)-f(\xi)\right)
\end{aligned}
$$

with $\xi^{i, j}$ the configuration obtained from $\xi$ by removing a particle from $i$ and putting it at $j$. Note that, in general, changing a representation does not imply that a generator continues to be a generator: the fact that $H$ and $H_{\text {dual }}$ are well-defined as a Hamiltonian is conserved by 
similarity transformations (change of representation) but their property of being (minus) the adjoint of the generator of a Markov process is dependent on the representation, and needs to be verified by hand.

\subsection{The Duality Function Explained}

In [6] we found that $L$ and $L_{\text {dual }}$ are dual processes, with duality function

$$
D(x, \xi)=\prod_{i \in \mathcal{S}} D_{i}\left(x_{i}, \xi_{i}\right)
$$

with

$$
D_{i}\left(x_{i}, \xi_{i}\right)=\frac{x_{i}^{2 \xi_{i}}}{\left(2 \xi_{i}-1\right) ! !}
$$

We now show how these functions arise from the change of representation. Suppose that we find a function

$$
C=C(x, \xi)=\prod_{i \in \mathcal{S}} C_{i}\left(x_{i}, \xi_{i}\right)
$$

such that

$$
K_{i}^{a} C_{i}=C_{i} \mathcal{K}_{i}^{a}
$$

for $a \in\{+,-, 0\}, i \in \mathcal{S}$ and $K_{i}^{a}$, resp. $\mathcal{K}_{i}^{a}$ defined in (70), (73). The "matrix product" in the 1.h.s. of (79) is defined as the differential operator $K_{i}^{a}$ working on the $x_{i}$-variable of $C_{i}\left(x_{i}, \xi_{i}\right)$, and in the r.h.s. $C_{i} \mathcal{K}_{i}^{a}\left(x_{i}, \xi_{i}\right)=\sum_{\xi_{i}^{\prime}} C\left(x_{i}, \xi_{i}^{\prime}\right) \mathcal{K}_{i}^{a}\left(\xi_{i}^{\prime}, \xi_{i}\right)$.

Then for the generators $L$ in (68), (69) and the generator $L_{\text {dual }}$ in (75), (76) we find, as a consequence of (79) and using that $L^{*}=L$,

$$
L C=L^{*} C=C L_{d u a l}^{*}
$$

i.e., such a function $C$ is a duality function (cfr. (6)).

The equation (79) is most easy of $a=+$, it then reads

$$
\frac{1}{2} x_{i}^{2} C_{i}\left(x_{i}, \xi_{i}\right)=\left(\frac{1}{2}+\xi_{i}\right) C_{i}\left(x_{i}, \xi_{i}+1\right)
$$

To find $C_{i}\left(x_{i}, 0\right)$ we use $K_{i}^{-} C_{i}\left(x_{i}, 0\right)=0$ (that follows from (73), (79)) so we can choose $C_{i}\left(x_{i}, 0\right)=1$ and then find, via $(81)$

$$
C_{i}\left(x_{i}, \xi_{i}\right)=\frac{x_{i}^{2 \xi_{i}}}{\left(2 \xi_{i}-1\right) ! !}
$$

which is exactly the duality function that we found in [6]. It is then easy to verify that (79) is also satisfied for $a \in\{-, 0\}$ with the choice (82).

\subsection{Reversible Measures of the Dual-BEP}

The dual of the BEP, with generator $L_{\text {dual }}$ in (75) and (76), is in itself an interacting particle system (particles attract each other), and it can therefore be considered as a model of 
independent interest. In some sense, it can be viewed as "the bosonic counterpart" of the SEP. Surprisingly, despite the interaction, the process has reversible product measures, as is shown below. Remark that, due to the attractive interaction between the particles, this process does not fall in the class of "misantrope processes" considered in [1, 3] (where one also has in particular cases stationary product measures, despite interaction).

Theorem 5.1 Consider, for $\lambda<1 / 2$ the translation invariant product measure $v_{\lambda}$ on $\mathbb{N}^{\mathcal{S}}$ with marginals

$$
v_{\lambda}\left(\eta_{0}=k\right)=\frac{1}{Z_{\lambda}} \frac{(2 k-1) ! !}{k !} \lambda^{k}
$$

where the normalization is

$$
Z_{\lambda}=\sum_{k=0}^{\infty} \frac{(2 k-1) ! !}{k !} \lambda^{k}=\frac{1}{\sqrt{(1-2 \lambda)}}
$$

Then $v_{\lambda}$ is reversible for the process with generator $L_{\text {dual }}$ in (75) and (76).

Proof From the generator (75), (76), we infer that

$$
\alpha_{i} \alpha_{j} 2 i(2 j+1)=2(j+1)(2 i-1) \alpha_{j+1} \alpha_{i-1}
$$

is a sufficient condition for detailed balance of a product measure $\mu$ with marginals $\mu\left(\eta_{0}=k\right)=\alpha_{k}$ for the generator $L_{i j}^{\text {dual }}$, which is sufficient for detailed balance for $L_{d u a l}$. This leads to

$$
\frac{\alpha_{j+1}}{\alpha_{j}}=\frac{2 j+1}{2 j+2}(2 c)
$$

for some positive constant $c$. This in turn gives

$$
\alpha_{j}=\frac{(2 j-1) ! !}{j !}(2 c)^{j} \alpha_{0}
$$

which is (83). The explicit expression for $Z_{\lambda}$ can be obtained easily from the identity

$$
\int_{-\infty}^{\infty} x^{2 k} e^{-x^{2} / 2} d x=\sqrt{2 \pi}(2 k-1) ! !
$$

\section{The Brownian Energy Process}

As it was done for SEP, it is interesting to consider the Brownian Momentum Process on ladder graph $\mathcal{S} \times \mathcal{I}$ with $|\mathcal{I}|=m \in \mathbb{N}$ levels and look at the induced process which gives the energy at each site.

\subsection{Generator}

Consider the generator, working on local functions $f: \mathbb{R}^{\mathcal{S} \times \mathcal{I}} \rightarrow \mathbb{R}$

$$
L=\sum_{i, j \in \mathcal{S}} \sum_{\alpha, \beta=1}^{m} p(i, j) L_{i \alpha, j \beta}
$$


where

$$
L_{i \alpha, j \beta}=\left(x_{i, \alpha} \frac{\partial}{\partial x_{j, \beta}}-x_{j, \beta} \frac{\partial}{\partial x_{i, \alpha}}\right)^{2}
$$

In this section we show that for the process with the generator above, the total energy per site defined via

$$
z_{i}=\sum_{\alpha=1}^{m} x_{i, \alpha}^{2}
$$

is again a Markov process

Theorem 6.1 Consider the process $x(t)=\left(x_{i, \alpha}(t)\right)_{i \in \mathcal{S}, \alpha=1, \ldots, m}$ with generator $L$ of (85) and (86). Consider the corresponding process $z(t)=\left(z_{i}(t)\right)_{i \in \mathcal{S}}$ defined via the mapping (87). This is a Markov process on $\mathbb{R}_{+}^{\mathcal{S}}$ with generator

$$
L^{(m)}=\sum_{i j \in \mathcal{S}} p(i, j) L_{i j}^{(m)}
$$

with

$$
L_{i j}^{(m)}=4 z_{i} z_{j}\left(\frac{\partial}{\partial z_{i}}-\frac{\partial}{\partial z_{j}}\right)^{2}-2 m\left(z_{i}-z_{j}\right)\left(\frac{\partial}{\partial z_{i}}-\frac{\partial}{\partial z_{j}}\right)
$$

and with stationary measures which are product measures with chi-squared marginals.

Proof Denote $\pi:\left(x_{i, \alpha}\right)_{i \in \mathcal{S}, \alpha=1, \ldots, m} \mapsto\left(z_{i}\right)_{i \in \mathcal{S}}$. Denote by $\partial_{i}$ partial derivative w.r.t. $z_{i}$ and by $\partial_{i, \alpha}$ partial derivative w.r.t. $x_{i, \alpha}$. Then, using the identities

$$
\begin{aligned}
\partial_{i, \alpha} & =2 x_{i, \alpha} \partial_{i} \\
\partial_{i, \alpha}^{2} & =2 \partial_{i}+4 x_{i \alpha}^{2} \partial_{i}^{2} \\
\partial_{i, \alpha} \partial_{j, \beta} & =4 x_{i, \alpha} x_{j, \beta} \partial_{i} \partial_{j}
\end{aligned}
$$

we find for a function $f: \mathbb{R}^{\mathcal{S} \times \mathcal{I}} \rightarrow \mathbb{R}$ depending on $x$ only through $z=\pi(x)$

$$
L f \circ \pi(x)=\left(L^{(m)} f\right)(\pi(x))
$$

The proof then proceeds via the martingale problem as in the proof of Theorem 4.2. The stationary measure of the process with generator $L^{(m)}$ is deduced from the knowledge of the stationary measure for the process with generator $L$. Indeed, it is easy to check that for the process $x_{i, \alpha}(t)$, products of Gaussian measures $\bigotimes_{i \in \mathcal{S}_{1}, \alpha} N\left(0, \sigma^{2}\right)$ are invariant and ergodic. The image measure under the transformation $\pi(x)=z$ are products $\bigotimes_{i \in \mathcal{S}_{1}} \chi_{m}^{2}(\sigma)$ where for $\sigma=1, \chi_{m}^{2}(1)$ is the chi-squared distribution with $m$ degrees of freedom, and for general $\sigma$, follows from scaling $\chi_{m}^{2}\left(\sigma^{2}\right)=\sigma^{2} \chi_{m}^{2}(1)$.

\subsection{Duality}

In this section we show that the BEP defined above has a dual process which is again a jump process. 
To construct the dual we follow a procedure similar to the one of the previous section. Remark that the generator $L$ in (85) and (86) can be written in terms of the operators

$$
K_{i}^{a,(m)}=\sum_{\alpha=1}^{m} K_{i, \alpha}^{a}
$$

with $a \in\{+,-, 0\}$, where

$$
\begin{aligned}
K_{i, \alpha}^{+} & =\frac{1}{2} x_{i, \alpha}^{2} \\
K_{i, \alpha}^{-} & =\frac{1}{2} \frac{\partial^{2}}{\partial x_{i, \alpha}^{2}} \\
K_{i, \alpha}^{0} & =\frac{1}{4}\left(\frac{\partial}{\partial x_{i, \alpha}}\left(x_{i, \alpha} \cdot\right)+x_{i, \alpha} \frac{\partial}{\partial x_{i, \alpha}}\right)
\end{aligned}
$$

In other words $L$ is related to a quantum spin chain with Hamiltonian

$$
H^{(m)}=-L^{*}=-4 \sum_{i j \in \mathcal{S}} p(i, j)\left(K_{i}^{+,(m)} K_{j}^{-,(m)}+K_{i}^{-,(m)} K_{j}^{+,(m)}-2 K_{i}^{0,(m)} K_{j}^{0,(m)}+\frac{m^{2}}{8}\right)
$$

where the $K$-operators in (92) and (93) satisfy the commutation relations of $S U(1,1)$. Moreover the $K$ operators defined in (92), (93), can be rewritten in $z$-variables as follows:

$$
\begin{aligned}
K_{i}^{+,(m)} & =\frac{1}{2} z_{i} \\
K_{i}^{-(m)} & =2 z_{i} \partial_{i}^{2}+m \partial_{i} \\
K_{i}^{0,(m)} & =z_{i} \partial_{i}+\frac{m}{4}
\end{aligned}
$$

The generator $L^{(m)}$ of (88) is then simply minus the adjoint of the Hamiltonian $H^{(m)}$ in (94), rewritten with $K$-operators in $z$-variables.

At this point it is important to remark that the $S U(1,1)$ group admits a family of discrete infinite dimensional discrete representation labeled by $m \in \mathbb{N}$ given by

$$
\begin{aligned}
\mathcal{K}_{i}^{+,(m)}\left|\xi_{i}\right\rangle & =\left(\frac{m}{2}+\xi_{i}\right)\left|\xi_{i}+1\right\rangle \\
\mathcal{K}_{i}^{-,(m)}\left|\xi_{i}\right\rangle & =\xi_{i}\left|\xi_{i}-1\right\rangle \\
\mathcal{K}_{i}^{0,(m)}\left|\xi_{i}\right\rangle & =\left(\frac{m}{4}+\xi_{i}\right)\left|\xi_{i}\right\rangle
\end{aligned}
$$

where $i \in \mathcal{S}$ and $\xi_{i} \in \mathbb{N}$ and $|0\rangle,|1\rangle,|2\rangle, \ldots$ denotes the canonical basis on $l_{2}(\mathbb{N})$. We then define a new generator via the same Hamiltonian as in (94), but now in the representation (96):

$$
H_{\text {dual }}^{(m)}=-L_{\text {dual }}^{*}=-4 \sum_{i j \in \mathcal{S}} p(i, j)\left(\mathcal{K}_{i}^{+,(m)} \mathcal{K}_{j}^{-,(m)}+\mathcal{K}_{i}^{-,(m)} \mathcal{K}_{j}^{+,(m)}-2 \mathcal{K}_{i}^{0,(m)} \mathcal{K}_{j}^{0,(m)}+\frac{m^{2}}{8}\right)
$$


Using the representation (96) we deduce that the Hamiltonian above defines a Markov process $\left(\xi_{t}\right)_{t \geq 0}$ with state space $\mathbb{N}^{\mathcal{S}}$ and generator

$$
L_{\text {dual }}^{(m)}=\sum_{i, j \in \mathcal{S}} p(i, j) L_{i j}^{(m), \text { dual }}
$$

where

$$
\begin{aligned}
L_{i j}^{(m), \text { dual }} f(\xi)= & 2 \xi_{i}\left(2 \xi_{j}+m\right)\left(f\left(\xi^{i, j}\right)-f(\xi)\right) \\
& +2 \xi_{j}\left(2 \xi_{i}+m\right)\left(f\left(\xi^{j, i}\right)-f(\xi)\right)
\end{aligned}
$$

The duality function are given in the following theorem.

Theorem 6.2 The processes with generator $L^{(m)}$ and $L_{d u a l}^{(m)}$ are each others dual, with duality function

$$
D(z, \xi)=\prod_{i \in \mathcal{S}} D_{i}\left(z_{i}, \xi_{i}\right)
$$

where

$$
D_{i}\left(z_{i}, \xi_{i}\right)=z_{i}^{\xi_{i}} \frac{\Gamma\left(\frac{m}{2}\right)}{2 \xi_{i} \Gamma\left(\frac{m}{2}+\xi_{i}\right)}
$$

with $\Gamma(t)=\int_{0}^{\infty} x^{t-1} e^{-x} d x$ the gamma function.

Proof Let

$$
C_{i}\left(z_{i}, \xi_{i}\right)=z_{i}^{\xi_{i}} \frac{\Gamma\left(\frac{m}{2}\right)}{2^{\xi_{i}} \Gamma\left(\frac{m}{2}+\xi_{i}\right)}
$$

One verifies easily that

$$
K_{i}^{a,(m)} C_{i}=C_{i} \mathcal{K}_{i}^{a,(m)}
$$

for $a=+,-, 0$. The proof then continues as in Sect. 5.3.

\subsection{The Instantaneous Thermalization Limit and the KMP Process}

In the KMP model, introduced in [8], the energies $E_{i}$ of different sites $i \in \mathcal{S}$ are updated by selecting a pair of lattice sites $(i, j)$ and uniformly redistributing the energy under the constraint of conserving $E_{i}+E_{j}$. In this section we show that the KMP model arises by taking what we call here an instantaneous thermalization limit of the process with generator $L^{(m)}$, for the case $m=2$.

We start by computing the stationary measure of the process with generator $L_{i j}^{(m)}$.

Lemma 6.3 Let $\left(z_{i}(t), z_{j}(t)\right)$ be the Markov process with generator $L_{i j}^{(m)}$, starting from an initial condition $\left(z_{i}(0), z_{j}(0)\right)$ with $z_{i}(0)+z_{j}(0)=E$. Then in the limit $t \rightarrow \infty$ the distribution of $\left(z_{i}(t), z_{j}(t)\right)$ converges to the distribution of the couple $((E+\epsilon) / 2,(E-\epsilon) / 2)$ where $\epsilon$ has probability density

$$
f(\epsilon)=C_{m}\left(E^{2}-\epsilon^{2}\right)^{\frac{m}{2}-1}
$$

$-E \leq \epsilon \leq E$ and $f=0$ otherwise, and where $C_{m}$ is the normalizing constant. 
Proof Define $(E(t), \epsilon(t))=\left(z_{i}(t)+z_{j}(t), z_{i}(t)-z_{j}(t)\right)$. Then simple rewriting of $L_{i j}^{(m)}$ in the new variables yields that $(E(t), \epsilon(t))$ is a Markov process with generator

$$
\mathcal{L}^{\prime}=4\left(E^{2}-\epsilon^{2}\right) \partial_{\epsilon}^{2}-4 m \epsilon \partial_{\epsilon}
$$

From the form of $\mathcal{L}^{\prime}$ we see immediately that $E$ is conserved and that for given $E, \epsilon(t)$ is an ergodic diffusion process with stationary measure solving

$$
\partial_{\epsilon}^{2}\left(4\left(E^{2}-\epsilon^{2}\right) f\right)+\partial_{\epsilon}(4 m \epsilon f)=0
$$

Now notice that the r.h.s. of (102) solves

$$
\partial_{\epsilon}\left(4\left(E^{2}-\epsilon^{2}\right) f\right)+(4 m \epsilon f)=0
$$

and hence (104).

Denote by $\gamma_{m}$ the distribution of $((E+\epsilon) / 2,(E-\epsilon) / 2)$. We can now define what me mean by instantaneous thermalization.

Definition 6.4 Let $f:[0, \infty)^{\mathcal{S}} \rightarrow \mathbb{R}$. For $e=\left(e_{i}\right)_{i \in \mathcal{S}}$ a configuration of energies, $(i, j) \in$ $\mathcal{S} \times \mathcal{S},\left(e_{i}^{\prime}, e_{j}^{\prime}\right) \in[0, \infty) \times[0, \infty)$ we denote by $t\left(e, e_{i}^{\prime}, e_{j}^{\prime}\right)$ the configuration obtained from $e$ by replacing $e_{i}$ by $e_{i}^{\prime}$ and $e_{j}$ by $e_{j}^{\prime}$. The instantaneous thermalization of a pair $(i, j) \in \mathcal{S} \times \mathcal{S}$ is defined by

$$
\mathcal{T}_{i j}^{(m)} f(e)=\int f\left(t\left(e, e_{i}^{\prime}, e_{j}^{\prime}\right)\right) d \gamma_{m}\left(e_{i}^{\prime}, e_{j}^{\prime}\right)
$$

The instantaneously thermalized version of the Brownian energy process is then defined as the process with generator

$$
\mathcal{L}_{m}^{I T} f(e)=\sum_{i j \in \mathcal{S}} p(i, j)\left(\mathcal{T}_{i j}^{(m)} f(e)-f(e)\right)
$$

This means that with rate $p(i j)$ a pair $(i, j) \in \mathcal{S} \times \mathcal{S}$ is chosen and the energy is instantaneously thermalized according to the measure $\gamma_{m}$. From (102) one sees that, for $m=2$, the uniform redistribution of the KMP model is recovered.

It is interesting to consider the dual of the instantaneous thermalization process for general $m \in \mathbb{N}$. From the previous discussion one knows that in the case $m=2$ this is just the dual of the KMP model. However, the model with generator has for general $m$ a dual with different duality functions as is shown in Theorem 6.6 below. To introduce this dual, we remind the reader that the Brownian energy process with generator $L^{(m)}$ is dual to the discrete particle jump process with generator $L_{d u a l}^{(m)}$. The following lemma gives the stationary measure of the dual BEP, which is needed in the construction of the instantaneous thermalized version of the dual BEP.

Lemma 6.5 Let $\left(k_{t}, l_{t}\right)$ evolve according to the generator $\mathcal{L}_{\text {dual }}^{i j}$, and suppose that initially $k_{0}+l_{0}=N$, then in the limit $t \rightarrow \infty,\left(k_{t}, l_{t}\right)$ converges in distribution to $((N+\Delta) / 2$, $(N-\Delta) / 2)$ where $\Delta$ has distribution $\mu$ on $\{-N,-N+2, \ldots, N\}$ with

$$
\frac{\mu(\Delta)}{\mu(\Delta-2)}=\frac{(N-\Delta+2)(N+\Delta-2+m)}{(N+\Delta)(N-\Delta+m)}
$$


In particular for $m=2,\left(k_{t}, l_{t}\right)$ converges to the uniform measure on the set $\{(a, b) \in$ $\{0, \ldots, N\}: a+b=N\}$.

Proof The process $\left(N_{t}, \Delta_{t}\right):=\left(k_{t}+l_{t}, k_{t}-l_{t}\right)$ performs transitions $(N, \Delta) \rightarrow(N, \Delta-2)$ at rate $\frac{1}{4}(N+\Delta)(N-\Delta+m)$ and $(N, \Delta) \rightarrow(N, \Delta+2)$ at rate $\frac{1}{4}(N-\Delta)(N+\Delta+m)$. The marginal $\Delta_{t}$ is then an irreducible continuous-time Markov chain on the set $\{-N,-N+$ $2, \ldots, N\}$, and hence has a unique stationary measure. Since it is a pure birth and death chain, this measure is also reversible. The recursion (107) then follows from detailed balance.

We denote by $\hat{\gamma}_{m}(k, l)$ the stationary distribution of Lemma 6.5. For $\xi \in \mathbb{N}^{\mathcal{S}}$, and $(i, j) \in$ $\mathcal{S} \times \mathcal{S},\left(\xi_{i}^{\prime}, \xi_{j}^{\prime}\right) \in \mathbb{N} \times \mathbb{N}$ we denote by $t\left(\xi, \xi_{i}^{\prime}, \xi_{j}^{\prime}\right)$ the configuration obtained from $\xi$ by replacing the value at $i$ by $\xi_{i}^{\prime}$ and at $j$ by $\xi_{j}^{\prime}$. We then define the dual thermalization by

$$
\mathcal{T}_{i j}^{\text {dual, }(m)} f(\xi)=\sum_{\xi_{i}^{\prime}, \xi_{j}^{\prime}: \xi_{i}^{\prime}+\xi_{j}^{\prime}=\xi_{i}+\xi_{j}} f\left(t\left(\xi, \xi_{i}^{\prime}, \xi_{j}^{\prime}\right)\right) \hat{\gamma}_{m}\left(\xi_{i}^{\prime}, \xi_{j}^{\prime}\right)
$$

and the dual instantaneously thermalized energy process as the process with generator

$$
\mathcal{L}_{\text {dual }}^{I T,(m)} f(\xi)=\sum_{i j \in \mathcal{S}}\left(\mathcal{T}_{i j}^{\text {dual },(m)} f(\xi)-f(\xi)\right)
$$

Theorem 6.6 Consider the instantaneously thermalized version of the Brownian energy process, with generator $\mathcal{L}_{m}^{I T}$. This process is dual to the process with generator $\mathcal{L}_{\text {dual }}^{I T,(m)}$ with duality function given by

$$
\begin{aligned}
D(e, \xi) & =\prod_{i} D_{i}\left(e_{i}, \xi_{i}\right) \\
D_{i}\left(e_{i}, \xi_{i}\right) & =e_{i}^{\xi_{i}} \frac{\Gamma(m / 2)}{2^{\xi_{i}} \Gamma\left(m / 2+\xi_{i}\right)}
\end{aligned}
$$

Proof By the duality result for the Brownian energy process, Theorem 6.2, we have for all $(i, j) \in \mathcal{S} \times \mathcal{S}$

$$
L_{i j}^{(m)} D(e, \xi)=L_{i j}^{(m), d u a l} D(e, \xi)
$$

therefore,

$$
\lim _{t \rightarrow \infty}\left(e^{t L_{i j}^{(m)}}-i d\right) D(e, \xi)=\lim _{t \rightarrow \infty}\left(e^{t L_{i j}^{(m), d u a l}}-i d\right) D(e, \xi)
$$

The result then follows from the definition of the processes, together with Lemmas 6.3 and 6.5.

\subsection{Limiting Processes as $m \rightarrow \infty$}

As it was done for the $2 j$-SEP, we study here the limiting behavior of the $m$-BEP process for large $m$.

Theorem 6.7 Consider the process $\left\{z_{t}^{(m)}: t \geq 0\right\}$ with generator $L^{(m)}$ and initial condition $z^{(m)} \in \mathbb{R}_{+}^{\mathcal{S}}$ and its dual $\left\{\xi_{t}^{(m)}: t \geq 0\right\}$ with generator $L_{\text {dual }}^{(m)}$ and initial condition $\xi^{(m)} \in \mathbb{N}^{\mathcal{S}}$. Suppose that, as $m \rightarrow \infty, z^{(m)} \rightarrow z \in \mathbb{R}_{+}^{\mathcal{S}}$ and $\xi^{(m)} \rightarrow \xi \in \mathbb{N}^{\mathcal{S}}$. Then: 
1. The process $\left\{z_{t / m}^{(m)}: t \geq 0\right\}$ converges to the process, $\left(z_{t}\right)_{t \geq 0}$ started from $z$, with generator

$$
\begin{aligned}
L & =\sum_{i j \in \mathcal{S}} p(i, j) L_{i j} \\
L_{i j} & =-2\left(z_{i}-z_{j}\right)\left(\frac{\partial}{\partial z_{i}}-\frac{\partial}{\partial z_{j}}\right)
\end{aligned}
$$

2. The process $\left\{\xi_{t / m}^{(m)}\right\}$ converges to a system of independent random walkers $\left(\xi_{t}\right)_{t \geq 0}$ started from $\xi$, with generator

$$
\begin{aligned}
& L_{\text {dual }}=\sum_{i j \in \mathcal{S}} p(i, j) L_{i j}^{\text {dual }} \\
& L_{i j}^{\text {dual }}=2 \xi_{i}\left(f\left(\xi^{i j}\right)-f(\xi)\right)+2 \xi_{j}\left(f\left(\xi^{j i}\right)-f(\xi)\right)
\end{aligned}
$$

3. The two limiting processes $\left(x_{t}\right)_{t \geq 0}$ and $\left(\xi_{t}\right)_{t \geq 0}$ above are each other dual, with duality function

$$
D(x, \xi)=\prod_{i \in \mathcal{S}} x_{i}^{\xi_{i}}
$$

Proof The proof of items 1 and 2 proceeds like in Theorem 4.3. For item 3 compare to example in Sect. 3.5.

\subsection{Boundary Driven Process}

In this last section we consider the $m$-BEP process in contact at its boundary to energy reservoirs of the Ornstein-Uhlenbeck type. A duality result for the Brownian Momentum Process with reservoirs was already proven in [6]. Here we generalize this result to the general Brownian energy process for arbitrary $m \in \mathbb{N}$. We start from the momentum process $\left\{\left(x(t)_{i, \alpha}\right): i \in \mathcal{S}, \alpha=1, \ldots, m, t \geq 0\right\}$ on a ladder graph with $m$ levels and all levels at sites $i \in \partial \mathcal{S}$ connected to a thermalizing Ornstein-Uhlenbeck process which parameter $T_{i}$, to be thought as the temperature. The generator reads

$$
\begin{aligned}
L= & \sum_{i, j \in \mathcal{S}} p(i, j) \sum_{\alpha, \beta=1}^{m}\left(x_{i, \alpha} \frac{\partial}{\partial x_{j, \beta}}-x_{j, \beta} \frac{\partial}{\partial x_{i, \alpha}}\right)^{2} \\
& +\sum_{i \in \partial \mathcal{S}} \sum_{\alpha=1}^{m} T_{i} \frac{\partial^{2}}{\partial x_{i, \alpha}^{2}}-x_{i, \alpha} \frac{\partial}{\partial x_{i, \alpha}}
\end{aligned}
$$

If we now consider the induced process $\left\{z_{i}(t): i \in \mathcal{S}, t \geq 0\right\}$ measuring the energy at each site via the map

$$
z_{i}=\sum_{\alpha=1}^{m} x_{i, \alpha}^{2}
$$

then, using the identities (90), we find the generator

$$
\begin{aligned}
L= & \sum_{i, j \in \mathcal{S}} p(i, j) 4 z_{i} z_{j}\left(\frac{\partial}{\partial z_{i}}-\frac{\partial}{\partial z_{j}}\right)^{2}-2 m\left(z_{i}-z_{j}\right)\left(\frac{\partial}{\partial z_{i}}-\frac{\partial}{\partial z_{j}}\right) \\
& +\sum_{i \in \partial \mathcal{S}} 2 T_{i}\left(m \frac{\partial}{\partial z_{i}}+2 z_{i} \frac{\partial^{2}}{\partial z_{i}^{2}}\right)-2 z_{i} \frac{\partial}{\partial z_{i}}
\end{aligned}
$$


Introducing as usual a set $\partial_{e} \mathcal{S}$ of sink sites and a bijection $i \mapsto i_{e}$ which associate each boundary site $i \in \partial \mathcal{S}$ to a sink site $i_{e} \in \partial_{e} \mathcal{S}$, we have the following duality theorem:

Theorem 6.8 Let $\left(z_{t}\right)_{t \geq 0}$ denote the boundary driven $m-B E P$ with generator (115). Then $\left(z_{t}\right)_{t \geq 0}$ is dual to the process $\left(\xi_{t}\right)_{t \geq 0}$ with generator

$$
\begin{aligned}
L_{\text {dual }} f(\xi)= & \sum_{i, j \in \mathcal{S}} p(i, j) 2 \xi_{i}\left(2 \xi_{j}+m\right)\left(f\left(\xi^{i, j}\right)-f(\xi)\right)+2 \xi_{j}\left(2 \xi_{i}+m\right)\left(f\left(\xi^{j, i}\right)-f(\xi)\right) \\
& +\sum_{i \in \partial \mathcal{S}} 2 \xi_{i}\left(f\left(\xi^{i, i_{e}}\right)-f(\xi)\right)
\end{aligned}
$$

with duality function given by

$$
D(z, \xi)=\prod_{i \in \partial_{e} \mathcal{S}} T_{i}^{\xi_{i}} \prod_{i \in \mathcal{S}} z_{i}^{\xi_{i}} \frac{\Gamma\left(\frac{m}{2}\right)}{2^{\xi_{i}} \Gamma\left(\frac{m}{2}+\xi_{i}\right)}
$$

Proof The bulk part of the duality function coincides with the one of Theorem 6.2; the boundary part is easily checked with an explicit computation.

Open Access This article is distributed under the terms of the Creative Commons Attribution Noncommercial License which permits any noncommercial use, distribution, and reproduction in any medium, provided the original author(s) and source are credited.

\section{References}

1. Bahadoran, C., Guiol, H., Ravishankar, K., Saada, E.: A constructive approach to Euler hydrodynamics for attractive processes. Application to $k$-step exclusion. Stoch. Process. Appl. 99, 1-30 (2002)

2. Bernardin, C., Olla, S.: Fourier's law for a microscopic model of heat conduction. J. Stat. Phys. 121, 271 (2005)

3. Cocozza, C., Kipnis, C.: Processus de vie et de mort sur $R$ ou $Z$, avec interaction selon les particules les plus proches. C. R. Acad. Sci. Paris Sér. A-B 284, 1291 (1977)

4. Ethier, S.N., Kurtz, T.G.: Markov Processes. Characterization and Convergence. Wiley, New York (1986)

5. Giardina, C., Kurchan, J.: The Fourier law in a momentum-conserving chain. J. Stat. Mech., P05009 (2005)

6. Giardina, C., Kurchan, J., Redig, F.: Duality and exact correlations for a model of heat conduction. J. Math. Phys. 48, 033301 (2007)

7. Gwa, L.H., Spohn, H.: Bethe solution for the dynamic-scaling exponent of the noisy Burgers-equation. Phys Rev. A 46(2), 844-854 (1992)

8. Kipnis, C., Marchioro, C., Presutti, E.: Heat flow in an exactly solvable model. J. Stat. Phys. 27(1), 65-74 (1982)

9. Liggett, T.M.: Interacting Particle Systems. Classics in Mathematics. Classics in Mathematics. Springer, Berlin (2005). Reprint of the 1985 original

10. Möhle, M.: The concept of duality and applications to Markov processes arising in neutral population genetics models. Bernoulli 5(5), 761-777 (1999)

11. Sandow, S.: Partially asymmetric exclusion process with open boundaries. Phys. Rev. E 50(4), 26602667 (1994)

12. Sandow, S., Schütz, G.: On $U_{q}[S U(2)]$ symmetrical driven diffusion. Europhys. Lett. 26(1), 7-12 (1994)

13. Schütz, G., Sandow, S.: Non-Abelian symmetries of stochastic processes: Derivation of correlation functions for random-vertex models and disordered-interacting-particle systems. Phys. Rev. E 49, 2726-2741 (1994)

14. Schütz, G.: Duality relations for asymmetric exclusion processes. J. Stat. Phys. 86(5-6), 1265-1287 (1997) 
15. Schütz, G.: Exactly solvable models for many-body systems far from equilibrium. In: Domb, C., Lebowitz, J. (eds.) Phase Transition and Critical Phenomena, vol. 19, pp. 1-251. Academic Press, San Diego (2001)

16. Schütz, G.: J. Stat. Phys. 86(5-6), 1265-1287 (1997)

17. Spitzer, F.: Interaction of Markov processes. Adv. Math. 5, 246-290 (1970)

18. Spohn, H.: Long range correlations for stochastic lattice gases in a non-equilibrium steady state. J. Phys. A 16, 4275-4291 (1983)

19. Stroock, D.W., Varadhan, S.R.S.: Multidimensional Diffusion Processes. Classics in Mathematics. Springer, Berlin (2006). Reprint of the 1997 edition

20. Taussky, O., Zassenhaus, H.: On the similarity transformation between a matrix and its transpose. Pac. J. Math. 9(3), 893-896 (1959) 